\title{
Title: Microglia monitor and protect neuronal function via specialized somatic purinergic junctions
}

Short title: Microglia control neurons at somatic junctions

\author{
Authors: Csaba Cserép ${ }^{1, \dagger}$, Balázs Pósfai ${ }^{1,12, \dagger}$, Nikolett Lénárt ${ }^{1}$, Rebeka Fekete ${ }^{1,12}$, Zsófia I. László2,12, Zsolt \\ Lele ${ }^{2}$, Barbara Orsolits ${ }^{1}$, Gábor Molnár ${ }^{3}$, Steffanie Heindl ${ }^{4}$, Anett D. Schwarcz ${ }^{1}$, Katinka Ujvári ${ }^{1}$, Zsuzsanna \\ Környei ${ }^{1}$, Krisztina Tóth ${ }^{1,12}$, Eszter Szabadits ${ }^{1}$, Beáta Sperlágh ${ }^{5}$, Mária Baranyi ${ }^{5}$, László Csiba ${ }^{6}$, Tibor \\ Hortobágyi ${ }^{7}, Z s o ́ f i a ~ M a g l o ́ c z k y{ }^{8}$, Bernadett Martinecz ${ }^{1}$, Gábor Szabó ${ }^{9}$, Ferenc Erdélyi ${ }^{9}$, Róbert Szipőcs ${ }^{10}$, \\ Michael M. Tamkun ${ }^{11}$, Benno Gesierich4, Marco Duering ${ }^{4,13}$, István Katona², Arthur Liesz ${ }^{4,13}$, Gábor \\ Tamás ${ }^{3}$, Ádám Dénes ${ }^{1, *}$
}

\footnotetext{
Affiliations:

1 "Momentum" Laboratory of Neuroimmunology, Institute of Experimental Medicine, Budapest, Hungary.

2 “Momentum” Laboratory of Molecular Neurobiology, Institute of Experimental Medicine, Budapest, Hungary.

3 MTA-SZTE Research Group for Cortical Microcircuits of the Hungarian Academy of Sciences, Department of Physiology, Anatomy and Neuroscience, University of Szeged, Szeged, Hungary.

${ }^{4}$ Institute for Stroke and Dementia Research, Ludwig-Maximilians-University, Munich, Germany.

${ }^{5}$ Laboratory of Molecular Pharmacology, Institute of Experimental Medicine, Budapest, Hungary.

6 MTA-DE Cerebrovascular and Neurodegenerative Research Group, Department of Neurology, University of Debrecen, Debrecen, Hungary.

${ }^{7}$ Institute of Pathology, Faculty of Medicine, University of Szeged, Szeged, Hungary.

${ }^{8}$ Human Brain Research Laboratory, Institute of Experimental Medicine, Budapest, Hungary.

${ }^{9}$ Medical Gene Technology Unit, Institute of Experimental Medicine, Budapest, Hungary.

${ }^{10}$ Institute for Solid State Physics and Optics of Wigner RCP, Budapest, Hungary.

${ }^{11}$ Department of Biomedical Sciences, Colorado State University, Fort Collins, Colorado, USA.

${ }^{12}$ Szentágothai János Doctoral School of Neuroscience, Semmelweis University, Budapest, Hungary.

${ }^{13}$ Munich Cluster for Systems Neurology (SyNergy), Munich, Germany

*Correspondence to: Dr. Ádám Dénes, Institute of Experimental Medicine, Szigony u. 43, 1083 Budapest, Hungary. Email: denes.adam@koki.mta.hu; Phone: +36209549149

† C.C. and B.P. contributed equally to this work
} 


\section{Abstract}

Microglia are the main immune cells in the brain with roles in brain homeostasis and neurological diseases. Mechanisms underlying microglia-neuron communication remain elusive. Here, we identified an interaction site between neuronal cell bodies and microglial processes in mouse and human brain. Somatic microglia-neuron junctions possessed specialized nanoarchitecture optimized for purinergic signaling. Activity of neuronal mitochondria was linked with microglial junction formation, which was induced rapidly in response to neuronal activation and blocked by inhibition of P2Y12 receptors (P2Y12R). Brain injury-induced changes at somatic junctions triggered P2Y12R-dependent microglial neuroprotection, regulating neuronal calcium load and functional connectivity. Thus, microglial processes at these junctions could potentially monitor and protect neuronal functions.

One-sentence summary: Neuronal somata possess specialized, pre-formed sites, through which microglia monitor their status and exert neuroprotection. 
Microglia are the main immunocompetent cells of the nervous system and their role in brain development and maintenance of proper neuronal function throughout life is widely recognized $(1,2)$. Changes in microglial activity are linked with major human diseases including different forms of neurodegeneration, stroke, epilepsy and psychiatric disorders $(3,4)$.

Microglia perform dynamic surveillance of their microenvironment via motile microglial processes that constantly interact with neurons $(5,6)$. However, the molecular mechanisms of bidirectional microglianeuron communication are unclear. To date, the majority of studies have focused on the interactions between microglial processes and synaptic elements, including axonal boutons and dendritic spines, which have commonly been perceived as the main form of interaction between microglia and neurons $(7,8)$. However, neurons are extremely polarized cells with a high degree of functional independence concerning metabolism and signal integration in their dendritic and axonal compartments (9-11). The large-scale structure of neurons (i.e. their cell body and axonal/dendritic branches) in the brain is relatively stable under most conditions. In comparison, small synaptic structures, such as dendritic spines and axonal boutons are often distant from neuronal cell bodies and are highly dynamic. Thus, the interactions between microglia and synapses may not fully explain how microglia are capable of monitoring and influencing the activity of neurons, or how early events of cellular injury in the perisomatic compartment are detected. This may be particularly relevant for the migration and differentiation of neural precursors, cell survival and programmed cell death, adult neurogenesis and the phagocytosis of damaged neuronal cell bodies (12-15). It is not understood how microglia could monitor neuronal status over years or even decades, and discriminate salvageable neurons from irreversibly injured cells mainly based on changes occurring at distant synaptic structures.

To understand the possible mechanisms of effective communication between microglia and neurons, we tested the hypothesis that specialized junctions on neuronal cell bodies may support the dynamic monitoring and assistance of neuronal function by microglia. 


\section{Microglial processes contact specialized areas of neuronal cell bodies in mouse and human brains}

To visualize microglia together with cortical neurons and to study microglia-neuron interactions in the intact brain in real-time, CX3CR1 ${ }^{+/ G F P}$ microglia reporter mice were electroporated in utero with $p C A G$ IRES-tdTomato plasmid (Fig. S1A). In vivo two-photon (2P) imaging revealed microglial processes contacting the cell bodies of cortical layer 2-3 neurons in the adult brain (Fig. 1A, B; Movie S1). Microglial processes preferentially returned to the same areas on the neuronal soma (observed in the case of 23 neurons out of 28 from 3 mice). The average lifetime of somatic microglia-neuron contacts was 25 min (some contacts persisting for more than 1 hour, Fig. S1B), while dendritic contacts had a significantly shorter lifetime of 7.5 min (Fig. 1C, $p=0.00035, n=26$ contacts from 3 mice), similar to that reported for synaptic contacts (16). Post-hoc confocal laser scanning microscopy (CLSM) and electron microscopic analysis further validated the direct interaction between microglial processes and the cell bodies of cortical pyramidal neurons (Fig. 1D; Fig. S1C-D), termed somatic microglial junctions. Similar interactions were present on well-characterized interneuron populations, namely type 3 vesicular glutamate transporter positive (VGluT3+) and parvalbumin-expressing (PV+) cells in the neocortex and the hippocampus (Fig. S1E). Somatic microglia-neuron junctions were also observed in the human neocortex (Fig. 1E). Somatic microglial junctions were present on $93 \%$ of cortical pyramidal cells, $95 \%$ of vGluT3+, and $89 \%$ of PV+ interneurons in mice ( $n=443$ cells from 4 mice). Despite the well-established microglial regulation of neuronal synapses, only $9 \%$ of glutamatergic and $11 \%$ of GABAergic synapses were associated with microglial processes (Fig. 1F; Fig. S1F, n=1183 synapses from 4 mice). $87 \%$ of neurons in the human neocortex received microglial contact onto their cell body (Fig. 1E-F, n=170 cells from 3 patients). We also tested the possible presence of somatic microglial junctions in subcortical areas. $98 \%$ of neurons in the caudate putamen, $91 \%$ of neurons in the nucleus reticularis gigantocellularis and $96 \%$ of neurons in the medial septum were contacted by microglial processes ( $n=268$ cells from 2 mice). Thus, these contacts are evolutionary conserved and present in all main areas of the brain. 
Microglia at somatic junctions may sense changes in neuronal state via signals released by exocytosis. In neurons, clustered Kv2.1 proteins are well known to provide exocytotic surfaces via anchoring vesicle fusion molecules to the neuronal membrane $(17,18)$. Furthermore, both Kv2.1 and Kv2.2 proteins are involved in forming endoplasmic reticulum (ER)/plasma membrane (PM)-junctions (membrane trafficking hubs), and anchoring intracellular organelles to the neuronal plasma membrane (19). Microglia contacted neuronal somatic membranes at sites of Kv2.1/2.2 clustering (Fig. 1G). The integrated density of Kv2.1 signal at these sites was $96 \%$ higher, and the density of Kv2.2 signal was $254 \%$ higher compared to those without microglial contacts (Fig. $1 \mathrm{H}, \mathrm{p}<0.0001$ in both cases, $n=114$ and 107 from 3 mice). 87\% of all microglia-neuron contacts expressed both types of clusters, $6.3 \%$ expressed only Kv2.1, 4.5\% only Kv2.2 clusters, and only $1.8 \%$ of contacts were void of any Kv-clusters (Fig. 1I, $\mathrm{n}=111$ contacts from 2 mice). Furthermore, 99\% of neocortical and 94\% of hippocampal CA1-region neurons expressed both Kv2.1 and Kv2.2 channels at the cellular level (Fig. S2A-C). The spatial association between Kv2.1 clusters and microglial processes was also observed on human cortical neurons (Fig. S1G, H, n=21 cells). Because Kv2.1-clusters are implicated in a large number of cellular processes involved in cell-to-cell communication, we focused on Kv2.1 in our further experiments. Kv2.1 hot spots appeared to define pre-formed neuronal microdomains, because Kv2.1 clusters remained unaltered after selective elimination of microglia by PLX5622 (Fig. S1I, J, 4.71 cluster/cross section in control vs. 6.64 cluster/cs in depleted, $n=59$ cells from 4 mice). To test the functional involvement of Kv2.1-clusters in the formation of somatic junctions, we developed a dominant-negative Kv2.1 mutant construct (DNKv2.1). This construct could not integrate into the plasma membrane and blocked the forward trafficking of any endogenous Kv2 proteins that may be expressed. We transfected HEK293cells, which naturally lack Kv2.1 protein (20), with fluorescent protein-coupled Kv2.1 or DNKv2.1 constructs, and cocultured these with microglia. Microglial processes contacted Kv2.1-transfected HEK cells preferentially at Kv2.1 clusters, but not the DNKv2.1 transfected ones (Fig. 1J, Movie S2). 84\% of Kv2.1-transfected HEK cells received microglial process contacts (97\% of these contacts arrived onto 
Kv2.1-clusters), while only 5.4\% of DNKv2.1 transfected HEK cells received process contacts ( $\mathrm{n}=75$ cells from 3 experiments). Thus, cell surface expression and clustering of Kv2.1 proteins is sufficient to induce contact formation by microglial processes.

Activity-dependent exocytotic ATP or ADP release takes place from neuronal cell bodies under physiological conditions $(21,22)$. ATP (ADP) is a major chemoattractant for microglial processes via the microglial purinoceptor, $\mathrm{P} 2 \mathrm{Y} 12$ receptor $(\mathrm{P} 2 \mathrm{Y} 12 \mathrm{R})(5,23)$. We thus asked whether signaling via P2Y12R was also essential for microglia-neuron interactions at these somatic junctions. In fact, all microglia - but no other cells in the brain, including perivascular macrophages - were found to be P2Y12R-positive (Fig. S3), including their processes recruited to somatic junctions (Fig. S3B). The restriction of P2Y12R expression to microglia within the brain agrees with results of earlier single-cell transcriptomics studies $(24,25)$.

To investigate the nanoscale architecture of $\mathrm{P} 2 \mathrm{Y} 12 \mathrm{R}$ at somatic microglia-neuron junctions, we used correlated CLSM and STORM superresolution microscopy, which enables the precise assessment of P2Y12R and Kv2.1 clusters at $20 \mathrm{~nm}$ lateral resolution (26). P2Y12R formed dense clusters on microglial processes at somatic junctions, directly facing neuronal Kv2.1 clusters (Fig. 1K). Unbiased cluster analysis revealed that P2Y12R localization point (LP) density and cluster density were both significantly higher on microglial processes inside the junctions than on processes outside the junctions or on the whole microglial cell (Fig. 1K; Fig. S2D; for detailed statistics and numbers see Table S1). Furthermore, somatic contact-dependent clustering of P2Y12R occurred on both pyramidal cells and interneurons (Fig. S2E; for detailed statistics and numbers see Table S1). Contact-dependent molecular clustering, however, could not be observed in the case of the microglial calcium-binding protein, Iba1 (Fig. S2F). Contact-dependent P2Y12R clustering was specific to somatic junctions, immunogold density was $62 \%$ lower on microglial membranes contacting boutons than on ones contacting somata (Fig. S2G, $p=0.0002, n=26$ contacts from 3 mice). Thus, we suggest the existence of a functionally specialized, yet ubiquitous communication site between P2Y12R-positive microglial processes and neuronal cell bodies. 


\section{Somatic microglia-neuron junctions possess a unique nanoarchitecture and molecular fingerprints}

To further investigate the ultrastructural features of somatic microglia-neuron junctions, we performed transmission electron microscopy and high-resolution electron tomography with 3D reconstruction. P2Y12R immunogold labeling confirmed the formation of direct junctions between microglial processes and neuronal somata both in mice (Fig. 2A) and in postmortem human brain tissue (Fig. S4A). Microglianeuron junctions were composed of closely apposed mitochondria, reticular membrane structures, intracellular tethers and associated vesicle-like membrane structures within the neuronal cell body (Fig. 2A). 3D electron tomography confirmed this nano-architecture in neurons (Fig. 2B; Movie S3-4). These morphological features were not observed in perisomatic boutons contacted by microglia. Furthermore, automated 3D analysis of tomographic volumes showed that P2Y12R density negatively correlated with the distance between microglial and neuronal membranes within the junctions (Fig. 2C, D; Fig. S4C, $\mathrm{p}<0.001, \mathrm{n}=13055$ points from 3 contacts). We also compared P2Y12R density between microglial membrane surfaces establishing junctions with neuronal somata and adjacent surfaces (within a few $\mu \mathrm{m}-\mathrm{s}$ ) that contacted boutons or other neuronal elements. We detected a significantly higher P2Y12R density at microglial membranes directly contacting neuronal cell bodies (Fig. 2E; Movie S5, p=0.00115, $\mathrm{n}=24$ surfaces). This suggests an important role for purinergic signaling in the formation of somatic microglia-neuron junctions.

We also observed discrete intercellular structures in the extracellular space resembling cell adhesion molecules, connecting the membranes of microglia and neuronal cell bodies (average length $23.5 \pm 3.1$ $n m, n=89$ from 3 mice, Fig. S4B). This falls in the range of the size of integrins expressed by microglia (27, 28) or the width of immunological synapses between peripheral immune cells (29). Mitochondriaassociated membranes (MAM, average distance: $19.5 \mathrm{~nm}$; $\mathrm{n}=104$ from 3 mice, Fig. S4B) (30), and discrete tethers were also observed between mitochondria and MAM (Movie S4).

We hypothesized that mitochondrial ATP-production and changes in neuronal activity could trigger microglial process recruitment. Thus, we investigated the possible enrichment of neuronal mitochondria 
at microglial junctions on a large sample size, using an unbiased, semi-automatic analysis of the outer mitochondrial membrane protein TOM20. TOM20 immunofluorescent intensity was $420 \%$ higher at somatic junctions compared to adjacent areas (Fig. 2F, G, $p<0.001, n=14$ contacts from 2 mice), confirming the strong accumulation of neuronal mitochondria at the somatic junctions.

TOM20-containing vesicles were observed between mitochondria and the neuronal membrane in addition to TOM20-negative vesicles (Fig. 2H; Fig. S4E, F). This may suggest trafficking and possible exocytosis of mitochondria-derived vesicles (31) at somatic microglial junctions. Mitochondria-derived vesicles (MDVs) often integrate into the endo-lysosomal pathway (31), and these vesicles are positive for the lysosomal marker LAMP1 (32). Indeed, LAMP1-positive puncta were closely associated with 83.3\% of all Kv2.1 clusters at somatic junctions (Fig. S4G, $n=72$ contacts from 2 mice), suggesting the release of MDVs and lysosomal content at these junctions.

Kv2.1-immunogold clusters were tightly associated with the observed neuronal structures (i.e. closely apposed mitochondria, MAMs, ER, vesicle-like structures, cytoplasmatic densities) within these junctions (Fig. S4D). Similarly to our CLSM results (Fig. S1I), Kv2.1 nanoclustering was not affected by the absence of microglia (Fig. S4D). These structures may function as mitochondria-related signaling hubs in neurons that microglia can recognize. Vesicular release of mitochondria-derived ATP from neurons may occur in a vesicular nucleotide transporter (VNUT) dependent manner $(33,34)$. Indeed, vNUT signal intensity was 2.5 times higher in the vicinity of the neuronal membranes at somatic microglia-neuron junctions compared with areas outside the junctions (Fig. 2I, p=0.002, n=15 contacts from 2 mice). Neuronal vNUT labeling was concentrated between mitochondria and the microglia-contacted neuronal membranes (Fig. 2J).

Kv2.1 or vNUT signal was not present in perisomatic axon terminals (GABAergic synaptic boutons) including those contacted by microglial processes (Fig. S4H, I, n=220 boutons for Kv2.1, n=194 boutons for vNUT, from 2 mice), confirming again that these molecular fingerprints were associated with somatic microglia-neuron junctions. 
Physiological microglia-neuron communication at somatic junctions is P2Y12R-dependent and is linked with neuronal mitochondrial activity

Next, we aimed to test whether microglial process recruitment to somatic junctions was functionally linked with the activity of mitochondria in neurons. To this end, CX3CR1 ${ }^{+/ G F P}$ mice were electroporated in utero with the mitochondria-targeted CAG-Mito-R-Geco1 reporter construct (Fig. S5A). Again, we observed the involvement of somatic mitochondria in microglial junctions (Fig. 3A). In vivo 2P imaging was performed to monitor microglial process recruitment to neuronal mitochondria in the cerebral cortex (Fig. 3B). As expected, recruited microglial processes came to close apposition with neuronal mitochondria. These processes stayed in the vicinity of neuronal mitochondria for around 29 minutes in vivo (Fig. 3B, Movie S6, $n=25$ contacts on 19 neurons from 3 mice, median value), closely matching the value measured in tdTomato electroporated mice (Fig. 1C). To study the functional relationship between microglial junction formation and activity of neuronal mitochondria, we assessed intracellular changes of the metabolic electron carrier nicotinamide adenine dinucleotide (NADH) (35) in coronal slices of visual and somatosensory cortices from CX3CR1+/GFP mice. Intracellular NADH fluorescence showed a granular pattern indicating mitochondrial NADH source. Indeed, the NADH signal co-localized with MitoR-Geco1 signal, confirming its mitochondrial origin (Fig. S5C). To search for somatic junction formation, we performed $2 \mathrm{P}$ imaging, which allowed us to track the movement of microglial processes and monitor cytosolic NADH in viable layer 2/3 neurons simultaneously (Fig. S5D). We detected apparent increases in $\mathrm{NADH}$ intrinsic fluorescence (Fig. 3C, E, $p=0.024, n=10$ cells) parallel with the formation of somatic microglial junctions. In contrast, we found no changes in the mean intrinsic NADH fluorescence detected at neuronal somata contacted by microglial processes in $\mathrm{P} 2 \mathrm{Y} 12 \mathrm{R}^{-1-}$ tissue (Fig. 3D, E, p=0.3, $\mathrm{n}=11$ cells). Thus, microglial process recruitment to somatic junctions is linked to the metabolic activity of neuronal mitochondria via a P2Y12R-dependent mechanism. 
The molecular machinery and intercellular interactions identified above suggested the involvement of purinergic signaling in these somatic junctions. To test whether neuronal somata could release ATP at these sites, we conducted a series of in vitro experiments. Quinacrine-labeled ATP-containing vesicles localized between neuronal mitochondria and neuronal membranes were present at sites where microglial processes contacted neuronal Kv2.1 clusters in neuron-microglia cocultures (Fig. 3F). Quinacrine labeling also colocalized with vNUT signal (Fig. 3F), as previously demonstrated for neurons (33).

Next, we tested whether neuronal activity could release ATP-containing vesicles from neuronal cell bodies. $40 \mathrm{mM} \mathrm{KCl}$-stimulation induced a rapid membrane depolarization and calcium influx in cultured neurons (Fig. S5E, $n=23$ cells for FluoVolt measurements, $n=20$ cells for Rhod3 measurements). CLSM in vitro time-lapse imaging confirmed that quinacrine-labeled (ATP-containing) vesicles were released from neuronal cell bodies after $\mathrm{KCl}$-stimulation (Fig. 3G, H, 880\% increase in release events after $\mathrm{KCl}$ vs. $27 \%$ decrease after vehicle, $n=13$ cells). Size analysis confirmed that the smaller profiles were released (Fig. 3I, median diameter of released: $0.37 \mu \mathrm{m}$ and retained: $0.59 \mu \mathrm{m}, \mathrm{n}=118$ puncta, similarly to previous reports (33)). The larger ones were identified as mitochondria by their uniform TOM20-labeling (Fig. 3I, median diameter of vesicle-labeling: $0.45 \mu \mathrm{m}$ and mitochondrial-labeling: $1.2 \mu \mathrm{m}, \mathrm{n}=83$ puncta).

Next, we applied high sensitivity HPLC to detect the levels of released ATP in the medium (Fig $3 \mathrm{~J}$ ). $\mathrm{KCl}$ induced a robust ATP release in cultured neurons $(p=0.0218, n=11)$, which was not inhibited by a mixture of the synaptic calcium-channel blockers $\omega$-Agatoxin and $\omega$-Conotoxin $(S C, p=0.6532, n=11)$, but was almost completely inhibited by the L-type calcium channel blocker Nimodipine (known to be important for somatic vesicular release (36); NIM, $p=0.0271, n=10$ ), or the vNUT-inhibitor clodronate (CLO, $p=0.0284, n=10)$. These data confirmed the presence of an activity-dependent somatic ATP release from neurons. Because the main ligand for microglial P2Y12Rs is ADP, we tested the possible presence of nucleosidase expression at microglia-neuron contacts. Using CLSM and electron microscopy we found robust NTPDase1 expression on $99.6 \%$ of all microglial processes within the somatic junctions (Fig. 3K, 
$\mathrm{n}=275$ contacts from 2 mice). Thus, neuron-derived ATP can readily be converted into ADP, and sensed by microglia right within the somatic junctions.

Because microglial processes are in a position at the somatic junctions to sense neuronal activity, we further explored the signaling mechanisms at these sites in vivo, by $2 \mathrm{P}$ imaging in CX3CR1+/GFP microglia reporter mice that were electroporated in utero with the neuronal reporter pCAG-IRES-tdTomato (Fig. 4A, B). Administration of the potent and selective P2Y12R-inhibitor PSB0739 (PSB) into the cisterna magna (i.c.m.) reduced somatic junction lifetime by $45 \%$, but did not affect the lifetime of dendritic microglia-neuron contacts (Fig. 4 , ctrl som. vs. PSB som. $p=0.0331, n=40$ ). We also tested synapse density after acute i.c.m. administration of vehicle (control) or PSB. PSB treatment did not alter neocortical synapse numbers (Fig. S6G, 0.353 synapse $/ \mu \mathrm{m}^{2}$ in control, and 0.352 synapse/ $\mu \mathrm{m}^{2}$ in PSBinjected, $n=423$ appositions from 4 animals). Because the maintenance of somatic microglia-neuron junctions depends on physiological P2Y12R function, we tested whether microglia would react directly to changes in neuronal activity. We induced neuronal activation by using the chemogenetic DREADD (Designer Receptor Exclusively Activated by Designer Drug) approach. pAAV carrying the hSyn$\mathrm{hM} 3 \mathrm{D}(\mathrm{Gq})$-mCherry construct was injected into the cerebral cortex of $\mathrm{P} 2 \mathrm{Y} 12 \mathrm{R}^{+/+}$and $\mathrm{P} 2 \mathrm{Y} 12 \mathrm{R}^{-/-}$mice that had been crossed with CX3CR1 $1^{+/ G F P}$ mice to visualize microglial responses in the presence or absence of P2Y12R signaling (Fig. S5F, G). After intraperitoneal injection of clozapine-N-oxide (CNO) to induce $\mathrm{hM} 3 \mathrm{D}(\mathrm{Gq})-\mathrm{DREADD}$ activation, we observed a $234 \%$ increase in neuronal cFos signal compared to vehicle treatment (Fig. $\mathrm{S} 5 \mathrm{H}, \mathrm{p}<0.001, \mathrm{n}=100$ ), confirming a specific and robust neuronal activation.

Chemogenetic neuronal activation resulted in an increased microglial process coverage of the soma of DREADD- and cFos-coexpressing neurons in P2Y12R $\mathrm{R}^{+/+}$mice (Fig. 4D, 243\% of control, $p=0.0139, n=101$ neurons from 8 mice), but not in $\mathrm{P} 2 \mathrm{Y} 12 \mathrm{R}^{-1-}$ mice (Fig. 4E, 133\% of control, $\mathrm{p}=0.7497, \mathrm{n}=85$ neurons from 6 mice). We also tested the effect of acute central pharmacological blockade of microglial P2Y12Rs (Fig. 4F), and found that PSB injected i.c.m. completely abolished the neuronal activity-induced increase in microglial process coverage (Fig. 4G, 72.34\% lower process coverage in CNO+PSB than in CNO+vehicle, 
$\mathrm{p}<0.001, \mathrm{n}=124$ neurons from 6 mice). Thus, microglia dynamically react to changes in neuronal activity at somatic microglia-neuron junctions in a P2Y12R-dependent manner, leading to a rapid increase of somatic coverage by microglial processes.

\section{Microglia protect neurons after acute brain injury in a P2Y12R-dependent manner via altered somatic junctions}

Because somatic microglia-neuron junctions were abundant in the healthy brain, we next asked if these morpho-functional communication sites were altered in response to brain injury. Microglia are known to respond rapidly to changes in neuronal activity in the boundary zone of the infarct after stroke (37). Thus, we performed experimental stroke and delineated the evolving penumbra based on the metabolic activity of the tissue as assessed by the redox indicator TTC (tetrazolium chloride) coregistered with the immunofluorescent signal for MAP2 and microglia (Fig. S6A). We observed the fragmentation of mitochondria (Fig. 5A, 74\% decrease of individual mitochondrial area, $46 \%$ decrease of mitochondrial major axis, $\mathrm{p}<0.001$ for both, $\mathrm{n}=189$ mitochondria) and an almost complete declustering of Kv2.1 proteins in morphologically intact penumbral neurons (Fig. 5B; Fig. S6E, from 4 to 0 median cluster/cross section and from 0.0947 to 0 cluster/ $\mu \mathrm{m}$ in control and stroke, respectively, $\mathrm{p}<0.001, \mathrm{n}=58$ cells). These morphological changes were accompanied by a robust increase in the microglial process coverage of neuronal cell bodies, originating from somatic microglia-neuron junctions in both mice and human postmortem brain tissues (Fig. 5B-E, mouse: 3.8 -fold increase, $p<0.001, n=30$ neurons; human: 1.5 -fold increase, $\mathrm{p}=0.007, \mathrm{n}=249$ neurons). Acute i.c.m. administration of the P2Y12R-inhibitor PSB, or preventing mitochondrial injury by using the mitochondrial ATP-sensitive potassium (KATP) channel opener diazoxide (38), completely abolished stroke-induced increases in microglial process coverage around somatic junctions (Fig. 5D, ctrl vs. stroke: $p<0.001$, PSB ctrl vs. PSB stroke: $p=0.792$, diazo. ctrl vs. diazo. stroke: $p=0.053, n=140$ neurons). Viability of the examined neurons with increased microglial process coverage was confirmed by normal chromatin structure and membrane integrity (Fig. S6B, C). 
Transmission electron tomography also confirmed increased microglial process coverage and mitochondrial fragmentation of neurons (Fig. 5C).

To test the impact of P2Y12R-dependent microglial functions on neuronal viability in vivo, we investigated pharmacological inhibition of P2Y12R by injection of PSB i.c.m. prior to middle cerebral artery occlusion (MCAo). Inhibition of microglial P2Y12R prevented increases in microglial process coverage of neuronal cell bodies in the penumbra and altered functional connectivity in the brain as assessed by a widefield imaging approach in Thy1-GCaMP6s mice (Fig. 5F, G). An absence of P2Y12R signaling significantly increased the area of functional disconnection (global connectivity $<0.6$ ) in the ipsilateral hemisphere during ischemia, accompanied by a trend towards elevated neuronal calcium-load (Fig. 5F; Fig. S6F, p=0.0439, n=17 mice). Seed-based connectivity analysis revealed a significant increase in the contralateral sensory hindlimb area after reperfusion in PSB treated animals. Moreover, connectivity analysis of 14 functional areas revealed a substantial and widespread increase in connectivity strength in the absence of microglial P2Y12R signaling (Fig. 5G, $p=0.0077, n=7$ mice).

To examine the effect of $\mathrm{P} 2 \mathrm{Y} 12 \mathrm{R}$ inhibition at the single neuron level in the evolving ischemic penumbra in vivo, we investigated GCaMP6f-injected mice with 2P microscopy. In control mice, neuronal GCaMP6f signal remained unchanged for the first 90 minutes of reperfusion, while blockade of microglial P2Y12Rs with PSB resulted in a strong elevation in neuronal calcium load (Fig. $5 \mathrm{H}, \mathrm{p}<0.0001, \mathrm{n}=96$ neurons, 3 mice). This corroborated the findings obtained from the widefield imaging approach also at the cellular level. Furthermore, P2Y12R inhibition significantly increased lesion volume at $24 \mathrm{~h}$ reperfusion (Fig. 5I, $54 \%$ increase, $p=0.008, n=20$ mice) and resulted in worse neurological outcome (Fig. 5I, Bederson score, stroke: $1.7 \pm 0.26$; stroke+PSB: $2.5 \pm 0.224, p=0.033, n=20$ mice).

To investigate the duration of PSB effect in vivo, two-hour long imaging sessions were performed with 2P microscopy 1-3 hours and 24-26 hours after i.c.m. PSB-injection ( $\mathrm{n}=173$ contacts analyzed, from 3 mice). The lifetime of somatic junctions was significantly reduced by up to 3 hours following PSB administration (56.3\% of lifetime under baseline conditions, $p=0.0139$ ), while there was no effect 
observed one day later (93.8\% of lifetime under baseline conditions), suggesting an acute effect of i.c.m. PSB (Fig. S6H, I). The acute effect of PSB is also confirmed by the histological measurements performed 4 hours after MCAo (Fig. 5D). To verify that i.c.m. injected PSB only inhibited microglial P2Y12Rs but not those expressed by circulating platelets, we measured ADP-induced platelet activation in plasma samples $1 \mathrm{~h}$ after MCAo, when BBB injury is apparent $(37,39,40)$. ADP-induced increases in platelet CD62P were not altered in mice treated with i.c.m. PSB compared to vehicle-treated animals (Fig. S5J). Thus, disintegration of somatic microglia-neuron junctions after neuronal injury triggers increased microglial process coverage of the cell bodies of compromised but potentially viable neurons via P2Y12R and mitochondrial signaling. This could allow the initiation of protective microglial responses that limit brain injury.

\section{Discussion}

Here we describe a form of interaction between microglia and neurons. Under physiological conditions, somatic microglia-neuron junctions were present on the majority of neurons in both mice and humans. The junctions appeared to function as communication sites that are rapidly altered in response to brain injury. We propose that microglia constantly monitor neuronal status via these somatic junctions, allowing neuroprotective actions to take place in a targeted manner.

Sites of somatic junctions in neurons were preferentially and repeatedly contacted by microglia. Such interactions had much longer lifetimes compared to the microglial contacts targeting dendrites. In previous studies, the proximity between microglial cell bodies or processes with neuronal somata has been observed in zebrafish and mice $(41,42)$. However, the formation of direct membrane-tomembrane junctions, the molecular identity of neuronal membranes contacted, activity-dependent recruitment of microglial processes to neuronal cell bodies, the mechanisms of junction formation, and the function of somatic microglia-neuron interactions have not been addressed. Thus, we took advantage of cutting edge neuroanatomical approaches and discovered that somatic microglia-neuron 
junctions are characterized by unique ultrastructural and molecular composition. These morphological and molecular features are absent in perisomatic boutons contacted by microglia, suggesting that the main form of neuronal quality control by microglial processes is not mediated by interactions between microglia and perisomatic axon terminals.

Mitochondria are the primary energy generators in cells, playing fundamental roles in calcium homeostasis, intracellular signaling $(43,44)$, neuronal quality control $(45)$, and in determining cellular fate (46). While neuronal mitochondria are also considered as "immunometabolic hubs" involved in antigen presentation and the regulation of innate immune responses $(47,48)$, changes in mitochondrial function due to metabolic imbalance, oxidative stress, inflammation, cellular injury or cell death occur in most neuropathological states (49). Mitochondria-associated membranes (MAM) are also considered to be key integrators of metabolic and immunological signals, playing a central role in neurodegeneration and cell-fate decisions $(30,50,51)$. Thus, somatic mitochondria and MAMs are ideally positioned to report neuronal status to microglia and to mediate neuronal quality control. In line with this, we show that the recruitment of microglial processes to somatic junctions in the vicinity of neuronal mitochondria is linked with mitochondrial activity. This may indicate rapid sensing of mitochondrial activity-associated changes of neurons by microglial processes via the release of ATP and other mediators, or the impact of microglia-derived substances on neuronal activity and/or mitochondrial function at somatic junctions. Neurons can execute somatic ATP release via pannexin hemichannels, voltage-dependent anion channels or through activity-dependent vesicle exocytosis $(21,22,36)$. Vesicular nucleotide transporter (VNUT) is known to be responsible for somatic vesicular ATP-release in neurons (34). In fact, we demonstrated the enrichment of VNUT between neuronal mitochondria and the somatic membranes contacted by microglia, and using time-lapse imaging and HPLC measurements, we confirmed the presence of activity-dependent somatic ATP release from neurons that was blocked by VNUT inhibition. TOM20-positive mitochondria-derived vesicles and other vesicles were also observed within the neuronal cytoplasm at somatic microglia-neuron junctions, together with the 
enrichment of LAMP1-positive lysosomes, which could - together with the released ATP - provide a constant readout of neuronal activity and mitochondrial function as seen in neurons and other cells (31, 52). The strong enrichment of VNUT in these contacts, the existence of an activity- and vNUT-dependent somatic ATP-release, the presence of filamentous cytoplasmatic structures connecting vesicles to the core of the junction, the presence of TOM20 immunogold positive vesicles within the contacts attached to the neuronal plasma membrane, close association of neuronal lysosomes, and the massive accumulation and nanoscale clustering of exocytosis-promoting Kv2.1-proteins within these contact sites collectively indicate the convergence of multiple parallel vesicular exocytotic pathways at somatic microglia-neuron junctions.

Kv2.1 channels are major regulators of neuronal potassium levels. However, they tend to assemble into discrete clusters on the surface of neurons, where they do not function as ion channels, but provide sites for intensive membrane trafficking as exo- and endocytotic hubs $(17,18,53)$. Furthermore, $\operatorname{Kv2.1}$ clusters are known to induce stable ER-plasma membrane junctions (53), anchoring MAMs and mitochondria into these morpho-functional units, providing an ideal site for release of mitochondriaassociated messenger molecules (31). The functional importance of these interactions is confirmed by our results showing that Kv2.1-clusters on transfected HEK-cells readily induced the formation of microglial process-contacts to these clusters, which could not be observed on HEK-cells transfected with the dominant-negative mutant Kv2.1. Furthermore, microglial P2Y12R clusters were precisely aligned with neuronal Kv2.1 clusters at somatic junctions.

The activation of P2Y12Rs was mainly associated with injury or pathological states in previous studies, and was considered negligible for physiological microglial surveillance, based on ex vivo studies (54). Compared to normal extracellular ATP levels in the brain, high levels of ATP (1 mM) were shown to induce P2Y12R-dependent microglial recruitment, similarly to that seen during microglial phagocytosis or in models of synaptic plasticity, while microglial surveillance is considered to be P2Y12R-independent $(54,55)$. Our in vivo results refine this view and highlight the importance of compartment-dependent 
effects of P2Y12R on microglial process responses: PSB0739 significantly reduced somatic junction lifetime but did not affect the lifetime of dendritic microglia-neuron contacts, while it abolished microglial reactions to altered neuronal activity, confirming P2Y12R-dependence of microglial actions under physiological conditions. Furthermore, neuronal mitochondrial activity was also linked with physiological microglial $\mathrm{P} 2 \mathrm{Y} 12 \mathrm{R}$ activity at these junctions. It is also possible that $\mathrm{P} 2 \mathrm{Y} 12 \mathrm{R}$-mediated actions are more important for sustaining than for forming somatic junctions during the communication between neuronal somata and microglial processes. The contact-dependent clustering of P2Y12 receptors further confirms their involvement in physiological microglia-neuron interactions at somatic junctions. Blockade of microglial P2Y12R left cortical synapse numbers completely unchanged and contact-dependent nano-clustering of microglial P2Y12Rs was not seen when microglia contacted synaptic boutons. Thus, microglia-neuron interactions at these sites are not only P2Y12R-dependent, but are fundamentally different from those seen at synapses.

The failure of most neuroprotection trials in stroke and other brain diseases strongly indicates the importance of understanding the complexity of pathophysiological processes, including microglial actions. Potentially salvageable neurons around the infarct core may show metabolic activity up to 6-17 hours following stroke in patients and experimental animals $(56,57)$. Here, Kv2.1 declustering was observed in compromised neurons of the penumbra as early as 4 hours after brain injury, which paralleled mitochondrial fragmentation in neurons, and increased microglial process coverage around somatic microglia-neuron junctions. Thus, P2Y12R-dependent microglial actions protect neurons, while blockade of microglial P2Y12R signaling alone impaired cortical network function, increased calcium load, and the area of ischemia-induced disconnection within two hours following stroke (a clinically relevant time window). This increased brain injury, similarly to that seen after the complete and selective elimination of microglia (37). These protective microglia- and P2Y12R-mediated effects were linked with mitochondrial actions initiated upon neuronal injury, because the KATP channel opener diazoxide abolished the increases in microglial process coverage of neurons after stroke, similarly to the 
blockade of P2Y12R signaling. All these results unequivocally indicate that microglia continuously monitor neuronal status via somatic junctions, rapidly responding to neuronal changes and initiating neuroprotective actions.

We propose that healthy neurons may constitutively release ATP and other signaling molecules at these junctions, reflecting their "well-being" towards microglia. In turn, disintegration of these specialized morpho-functional hubs due to excitotoxicity, energy depletion or other noxious stimuli may trigger rapid and inherently protective microglial responses, leading to the restoration of neuronal function or isolation and phagocytosis of dying neurons, in case terminal neuronal injury occurs (55). Along with P2Y12R-mediated microglial process recruitment, it is likely that a broad range of signals is integrated at somatic microglial junctions through which microglia may sense products of neuronal exocytosis, changes in the cell membrane (e.g. apoptotic signals), and alter the duration of physical contact or initiate phagocytosis. The most important open questions include the clarification of additional signaling mechanisms (vesicular and non-vesicular) involved in neuron-to-microglia communication at these junctions, and the mechanisms of microglial neuroprotection (e.g. regulation of neuronal ion-fluxes, neuronal calcium dynamics or the metabolism of neuronal mitochondria). Because the role of microglianeuron somatic junctions in most brain diseases is completely unknown, microglia-neuron interactions through these sites may differ in different forms of acute and chronic neuropathologies. 


\section{References and Notes:}

1. M. S. Thion, F. Ginhoux, S. Garel, Microglia and early brain development: An intimate journey. Science. 362, 185-189 (2018).

2. K. Kierdorf, M. Prinz, Microglia in steady state. J. Clin. Invest. 127, 3201-3209 (2017).

3. M. W. Salter, B. Stevens, Microglia emerge as central players in brain disease. Nat. Med. 23, 1018-1027 (2017).

4. W. M. Song, M. Colonna, The identity and function of microglia in neurodegeneration. Nat. Immunol. 19, 1048-1058 (2018).

5. D. Davalos et al., ATP mediates rapid microglial response to local brain injury in vivo. Nat. Neurosci. 8, 7528 (2005).

6. A. Nimmerjahn, Resting Microglial Cells Are Highly Dynamic Surveillants of Brain Parenchyma in Vivo. Science (80-. ). 308, 1314-1318 (2005).

7. Y. Wu, L. Dissing-Olesen, B. A. MacVicar, B. Stevens, Microglia: Dynamic Mediators of Synapse Development and Plasticity. Trends Immunol. 36, 605-613 (2015).

8. L. Weinhard et al., Microglia remodel synapses by presynaptic trogocytosis and spine head filopodia induction. Nat. Commun. 9, 1228 (2018).

9. J.-M. Cioni, M. Koppers, C. E. Holt, Molecular control of local translation in axon development and maintenance. Curr. Opin. Neurobiol. 51, 86-94 (2018).

10. T. Misgeld, T. L. Schwarz, Mitostasis in Neurons: Maintaining Mitochondria in an Extended Cellular Architecture. Neuron. 96 (2017), pp. 651-666.

11. M. Terenzio, G. Schiavo, M. Fainzilber, Compartmentalized Signaling in Neurons: From Cell Biology to Neuroscience. Neuron. 96, 667-679 (2017).

12. J. Aarum, K. Sandberg, S. L. B. Haeberlein, M. A. A. Persson, Migration and differentiation of neural precursor cells can be directed by microglia. Proc. Natl. Acad. Sci. 100, 15983-15988 (2003).

13. M. Ueno et al., Layer $V$ cortical neurons require microglial support for survival during postnatal development. Nat. Neurosci. 16, 543-551 (2013).

14. J. L. Marín-Teva, M. A. Cuadros, D. Martín-Oliva, J. Navascués, Microglia and neuronal cell death. Neuron Glia Biol. 7, 25-40 (2011).

15. A. Sierra et al., Surveillance, Phagocytosis, and Inflammation: How Never-Resting Microglia Influence Adult Hippocampal Neurogenesis. Neural Plast. 2014, 1-15 (2014).

16. H. Wake, A. J. Moorhouse, S. Jinno, S. Kohsaka, J. Nabekura, Resting microglia directly monitor the functional state of synapses in vivo and determine the fate of ischemic terminals. J. Neurosci. (2009), doi:10.1523/JNEUROSCI.4363-08.2009.

17. E. Deutsch et al., Kv2.1 cell surface clusters are insertion platforms for ion channel delivery to the plasma membrane. Mol. Biol. Cell. 23, 2917-2929 (2012).

18. L. Feinshreiber, D. Singer-Lahat, U. Ashery, I. Lotan, Voltage-gated Potassium Channel as a Facilitator of Exocytosis. Ann. N. Y. Acad. Sci. 1152, 87-92 (2009).

19. M. Kirmiz, N. C. Vierra, S. Palacio, J. S. Trimmer, Identification of VAPA and VAPB as Kv2 ChannelInteracting Proteins Defining Endoplasmic Reticulum-Plasma Membrane Junctions in Mammalian Brain Neurons. J. Neurosci. 38, 7562-7584 (2018). 
20. B. Jiang, X. Sun, K. Cao, R. Wang, Endogenous Kv channels in human embryonic kidney (HEK-293) cells. Mol. Cell. Biochem. 238, 69-79 (2002).

21. A. Menéndez-Méndez et al., Specific Temporal Distribution and Subcellular Localization of a Functional Vesicular Nucleotide Transporter (VNUT) in Cerebellar Granule Neurons. Front. Pharmacol. 8, 951 (2017).

22. R. D. Fields, Nonsynaptic and nonvesicular ATP release from neurons and relevance to neuron-glia signaling. Semin. Cell Dev. Biol. 22, 214-9 (2011).

23. S. E. Haynes et al., The P2Y12 receptor regulates microglial activation by extracellular nucleotides. Nat. Neurosci. 9, 1512-1519 (2006).

24. Y. Zhang et al., An RNA-Sequencing Transcriptome and Splicing Database of Glia, Neurons, and Vascular Cells of the Cerebral Cortex. J. Neurosci. 34, 11929-11947 (2014).

25. O. Butovsky et al., Identification of a unique TGF- $\beta$-dependent molecular and functional signature in microglia. Nat. Neurosci. 17, 131-143 (2014).

26. B. Dudok et al., Cell-specific STORM super-resolution imaging reveals nanoscale organization of cannabinoid signaling. Nat. Neurosci. 18, 75-86 (2015).

27. I. D. Campbell, M. J. Humphries, Integrin Structure, Activation, and Interactions. Cold Spring Harb. Perspect. Biol. 3, a004994-a004994 (2011).

28. H. Akiyama, P. L. McGeer, Brain microglia constitutively express beta-2 integrins. J. Neuroimmunol. 30, 8193 (1990).

29. F. E. McCann et al., The size of the synaptic cleft and distinct distributions of filamentous actin, ezrin, CD43, and CD45 at activating and inhibitory human NK cell immune synapses. J. Immunol. 170, 2862-70 (2003).

30. G. Csordás, D. Weaver, G. Hajnóczky, Endoplasmic Reticulum-Mitochondrial Contactology: Structure and Signaling Functions. Trends Cell Biol. 28, 523-540 (2018).

31. A. Sugiura, G.-L. McLelland, E. A. Fon, H. M. McBride, A new pathway for mitochondrial quality control: mitochondrial-derived vesicles. EMBO J. 33, 2142-56 (2014).

32. V. Soubannier et al., A vesicular transport pathway shuttles cargo from mitochondria to lysosomes. Curr. Biol. (2012), doi:10.1016/j.cub.2011.11.057.

33. T. Ho et al., Vesicular expression and release of ATP from dopaminergic neurons of the mouse retina and midbrain. Front. Cell. Neurosci. 9, 389 (2015).

34. Y. Moriyama, M. Hiasa, S. Sakamoto, H. Omote, M. Nomura, Vesicular nucleotide transporter (VNUT): appearance of an actress on the stage of purinergic signaling. Purinergic Signal. 13, 387-404 (2017).

35. A. M. Brennan, J. A. Connor, C. W. Shuttleworth, NAD(P)H Fluorescence Transients after Synaptic Activity in Brain Slices: Predominant Role of Mitochondrial Function. J. Cereb. Blood Flow Metab. 26, 1389-1406 (2006).

36. X. Zhang, Y. Chen, C. Wang, L.-Y. M. Huang, Neuronal somatic ATP release triggers neuron-satellite glial cell communication in dorsal root ganglia. Proc. Natl. Acad. Sci. 104, 9864-9869 (2007).

37. G. Szalay et al., Microglia protect against brain injury and their selective elimination dysregulates neuronal network activity after stroke. Nat. Commun. 7, 11499 (2016).

38. J. O. Onukwufor, D. Stevens, C. Kamunde, Bioenergetic and volume regulatory effects of mitoKATP channel modulators protect against hypoxia-reoxygenation-induced mitochondrial dysfunction. J. Exp. Biol. 219, 2743-51 (2016).

39. F. Orsini et al., Mannose-Binding Lectin Drives Platelet Inflammatory Phenotype and Vascular Damage After Cerebral Ischemia in Mice via IL (Interleukin)-1 $\alpha$. Arterioscler. Thromb. Vasc. Biol. 38, 2678-2690 
(2018).

40. K. Bekő et al., Contribution of platelet P2Y12 receptors to chronic Complete Freund's adjuvant-induced inflammatory pain. J. Thromb. Haemost. 15, 1223-1235 (2017).

41. Y. Li, X.-F. Du, C.-S. Liu, Z.-L. Wen, J.-L. Du, Reciprocal regulation between resting microglial dynamics and neuronal activity in vivo. Dev. Cell. 23, 1189-202 (2012).

42. R. D. Stowell et al., Cerebellar microglia are dynamically unique and survey Purkinje neurons in vivo. Dev. Neurobiol. 78, 627-644 (2018).

43. C. N. Hall, M. C. Klein-Flügge, C. Howarth, D. Attwell, Oxidative phosphorylation, not glycolysis, powers presynaptic and postsynaptic mechanisms underlying brain information processing. J. Neurosci. 32, 8940$51(2012)$.

44. N. S. Chandel, Mitochondria as signaling organelles. BMC Biol. 12, 34 (2014).

45. E. I. Rugarli, T. Langer, Mitochondrial quality control: a matter of life and death for neurons. EMBO J. 31, 1336-49 (2012).

46. A. Kasahara, L. Scorrano, Mitochondria: from cell death executioners to regulators of cell differentiation. Trends Cell Biol. 24, 761-70 (2014).

47. D. Arnoult, F. Soares, I. Tattoli, S. E. Girardin, Mitochondria in innate immunity. EMBO Rep. 12, 901-910 (2011).

48. G. R. Bantug et al., Mitochondria-Endoplasmic Reticulum Contact Sites Function as Immunometabolic Hubs that Orchestrate the Rapid Recall Response of Memory CD8 + T Cells. Immunity. 48, 542-555.e6 (2018).

49. A. U. Joshi, D. Mochly-Rosen, Mortal engines: Mitochondrial bioenergetics and dysfunction in neurodegenerative diseases. Pharmacol. Res. 138, 2-15 (2018).

50. J. Rieusset, Mitochondria-associated membranes (MAMs): An emerging platform connecting energy and immune sensing to metabolic flexibility. Biochem. Biophys. Res. Commun. 500, 35-44 (2018).

51. R. Bravo-Sagua et al., Cell death and survival through the endoplasmic reticulum-mitochondrial axis. Curr. Mol. Med. 13, 317-29 (2013).

52. G.-L. McLelland, S. A. Lee, H. M. McBride, E. A. Fon, Syntaxin-17 delivers PINK1/parkin-dependent mitochondrial vesicles to the endolysosomal system. J. Cell Biol. 214, 275-91 (2016).

53. P. D. Fox et al., Induction of stable ER-plasma-membrane junctions by Kv2.1 potassium channels. J. Cell Sci. 128, 2096-105 (2015).

54. C. Madry et al., Microglial Ramification, Surveillance, and Interleukin-1 $\beta$ Release Are Regulated by the TwoPore Domain K + Channel THIK-1. Neuron. 97, 299-312.e6 (2018).

55. R. Fekete et al., Microglia control the spread of neurotropic virus infection via P2Y12 signalling and recruit monocytes through P2Y12-independent mechanisms. Acta Neuropathol. 136, 461-482 (2018).

56. G. Marchal et al., Prolonged persistence of substantial volumes of potentially viable brain tissue after stroke: a correlative PET-CT study with voxel-based data analysis. Stroke. 27, 599-606 (1996).

57. J. C. Baron, M. E. Moseley, For how long is brain tissue salvageable? Imaging-based evidence. J. Stroke Cerebrovasc. Dis. 9, 15-20 (2000).

58. X. Wang, C. Zhang, G. Szábo, Q.-Q. Sun, Distribution of CaMKIla expression in the brain in vivo, studied by CaMKIla-GFP mice. Brain Res. 1518, 9-25 (2013).

59. A. H. Meyer, I. Katona, M. Blatow, A. Rozov, H. Monyer, In vivo labeling of parvalbumin-positive interneurons and analysis of electrical coupling in identified neurons. J. Neurosci. 22, 7055-64 (2002). 
60. G. López-Bendito et al., Preferential origin and layer destination of GAD65-GFP cortical interneurons. Cereb. Cortex. 14, 1122-33 (2004).

61. H. Dana et al., Thy1-GCaMP6 Transgenic Mice for Neuronal Population Imaging In Vivo. PLoS One. 9, e108697 (2014).

62. K. L. Schaar, M. M. Brenneman, S. I. Savitz, Functional assessments in the rodent stroke model. Exp. Transl. Stroke Med. 2, 13 (2010).

63. J. B. Bederson et al., Rat middle cerebral artery occlusion: evaluation of the model and development of a neurologic examination. Stroke. 17, 472-6 (1986).

64. K. L. Singel et al., Mitochondrial DNA in the tumour microenvironment activates neutrophils and is associated with worse outcomes in patients with advanced epithelial ovarian cancer. Br. J. Cancer (2019), doi:10.1038/s41416-018-0339-8.

65. J. R. Kremer, D. N. Mastronarde, J. R. McIntosh, Computer visualization of three-dimensional image data using IMOD. J. Struct. Biol. 116, 71-6 (1996).

66. J. V. Cramer et al., In vivo widefield calcium imaging of the mouse cortex for analysis of network connectivity in health and brain disease. Neuroimage. 199, 570-584 (2019).

67. D. Hinkle, W. Wiersma, S. Jurs, Applied Statistics for the Behavioural Sciences (2003).

\section{Acknowledgements}

We thank László Barna and the Nikon Imaging Center at the Institute of Experimental Medicine (IEM) for kindly providing microscopy support, David Mastronarde at MCDB for his continuous help with IMOD software, and Solt Kovács from ETH Zurich for scripting analytic tools. We are also grateful to Norbert Hájos (IEM), Zoltán Nusser (IEM) and James Trimmer (University of California, Davis) for their support and useful comments. We thank the Department of Pathology, St. Borbála Hospital, Tatabánya, and the Human Brain Research Lab at the IEM for providing human brain tissue and Dóra Gali-Györkei and Richárd Rácz for their excellent technical assistance. We also thank Plexxikon for providing PLX5622 and Deltagen for the donation of $\mathrm{P} 2 \mathrm{Y} 12 \mathrm{R}^{-/}$mice. Funding: This work was supported by "Momentum" research grant from the Hungarian Academy of Sciences (LP2016-4/2016 to A.D.) and ERC-CoG 724994 (A.D.), by the János Bolyai Research Scholarship of the Hungarian Academy of Sciences (C.C., and N.L), the UNKP-19-3-I (B.P.) and UNKP-19-4 (C.C.) New National Excellence Program of the Ministry for Innovation and Technology. Additionally, this work was funded by H2020-ITN-2018-813294-ENTRAIN (A.D.), Hungarian Academy of Sciences (G.T.), the National Research, Development and Innovation Office of Hungary (GINOP-2.3.2-15-2016-00018, VKSZ-14-1-2015-0155, G.T.), the Ministry of Human 
Capacities, Hungary (grant 20391-3/2018/FEKUSTRAT, G.T.), by the German Research Foundation (FOR 2879) and ERC-StG 802305 to A.L.; I.K. was supported by "Momentum" research grant from the Hungarian Academy of Sciences (LP2013-54), Hungarian Scientific Research Fund (OTKA, K 116915) and National Research, Development and Innovation Fund (VKSZ_14-1-2015-0155); M.M.T. was supported by National Institutes of Health grant RO1GM109888; B.S. and Zs.M. was supported by the grants of National Research, Development and Innovation Office of Hungary (K116654 to B.S., K125436 to Zs.M.) and by National Brain Research Program (2017-1.2.1-NKP-2017-00002); G.M. was supported by Hungarian Scientific Research Fund (OTKA, K 128863); L.Cs. was supported by National Research, Development and Innovation Fund (GINOP-2.3.2-15-2016-00048-Stay Alive); R.S. was supported by Hungarian Scientific Research Fund (OTKA, K 129047). This work was supported by EFOP-3.6.3.-VEKOP16-2017-00009 from Semmelweis University. Authors contributions: C.C., B.P. and A.D. conceived the project. Surgery was performed by N.L. and A.D.; two-photon imaging was performed by R.F., immunohistochemistry and light microscopy was performed by C.C., B.P., A.D., B.O., A.D.S and E.S.; STORM microscopy was performed by B.O., Electron microscopy was performed by C.C., B.P., E.S. and A.D.S., electron tomography was performed by C.C. and B.P., in vitro NADH imaging was performed by G.M. under the supervision of G.T., plasmid engineering and in utero electroporation was performed by Z. L. and Z. I. L., in vitro cell culture transfection and experiments were performed by Zs.K., K.T. and Z.I.L.; virus injection was performed by R.F. and B.M., widefield calcium imaging was performed by S.H. under the supervision of A.L.; HPLC measurements were performed by M.B. under the supervision of B.S.; C.C., B.P., B.O., G.M., S.H., N.L., A.D.S., K.U., A.L., A.D. analyzed data, L.C., T.H., Z.M. G.S., M.M.T. and F.E. contributed with critically important clinical and neuropathological data and materials, R.S. optimized the laser-optical setup for dual wavelength in vitro $2 \mathrm{P}$ measurements, I.K., G.T. and A.L. provided resources and essential intellectual contribution, and revised the manuscript. A.D. obtained funding and supervised the project. C.C. B.P. and A.D. wrote the paper with input from all authors. Competing interests: The authors declare no competing interests. Data and materials availability: 
PLX5622 was obtained under a materials transfer agreement (MTA) with Plexxikon. All data is available in the main text or the supplementary materials.

\section{Supplementary Materials:}

Materials and Methods

Supplementary Figure legends

Figures S1-S6

Tables S1-S3

Movies S1-S7

References (58-67) 

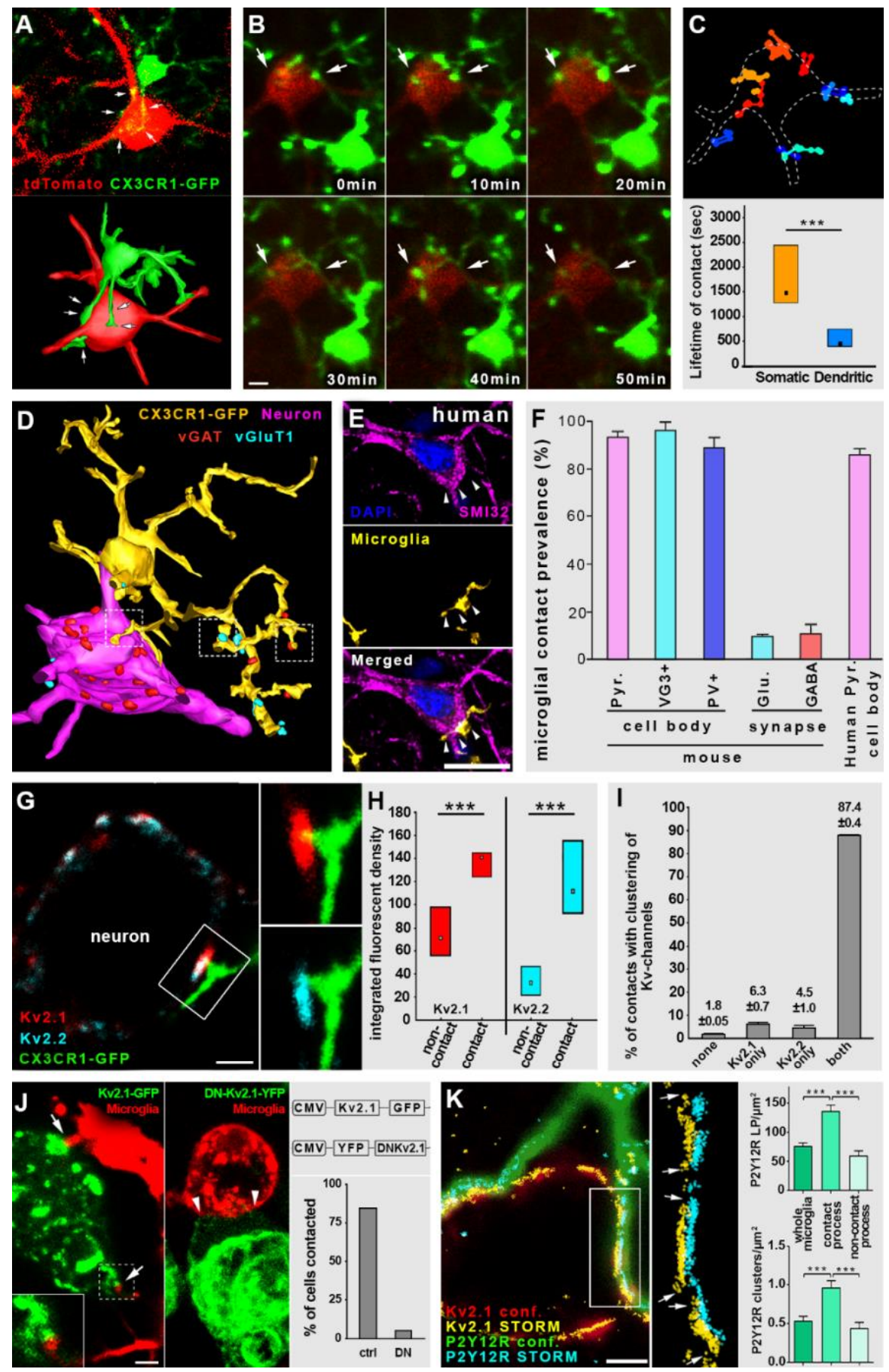

Figure 1. Microglia contact specialized areas of neuronal cell bodies in the mouse and the human brain

(A) Single image plane (upper panel) and 3D reconstruction (lower panel) from an in vivo 2-photon (2P) Z-stack shows a neocortical neuron (red) being contacted by microglial processes (green). (B) In vivo 2P time-lapse imaging shows temporal dynamics of microglia-neuron contacts. (C) Analyzed trajectories of microglial processes contacting the neuron on $B$. The lifetime of somatic contacts was significantly longer than dendritic ones. (D) 3D- 
reconstruction from high-resolution confocal laser scanning microscopy (CLSM) Z-stack shows that microglial processes (yellow) contact GABAergic (red) and glutamatergic (cyan) boutons as well as the neuronal cell body (Kv2.1 labeling, magenta). (E) CLSM images show P2Y12R+ microglial processes (yellow) contacting an SMI32+ neuronal cell body (magenta) in human neocortex. (F) Quantitative analysis of contact prevalence between microglial processes and different neuronal elements confirms that microglia contact the vast majority of neuronal cell bodies independently from neurochemical identity, while only a small fraction of synapses receive microglial contact. (G) CLSM image shows a neuronal cell body contacted by a microglial process at a KV2.1-2.2-cluster. (H) The integrated fluorescent density of both Kv2.1 and Kv2.2 signal is significantly higher within the contact site than elsewhere. (I) The vast majority of microglia-neuron junctions expressed both Kv2.1 and 2.2 clusters. (J) Microglial processes contact KV2.1-transfected HEK-cells at the clusters, but not those transfected with a dominant-negative mutant. (K) Overlaid images show microglial P2Y12R (green for CLSM and cyan for STORM) and neuronal KV2.1 (red and yellow) clusters overlapping. Arrows show borders of Kv2.1 clusters. P2Y12R clustering depends on the contact with neuronal cell body. Bar graphs show STORM localization point (LP) density (top) and density of identified P2Y12R clusters (bottom) on different parts of microglia (for statistics, see Table S1).

For statistical details, see supplementary text for Fig. 1. and Table S1. 

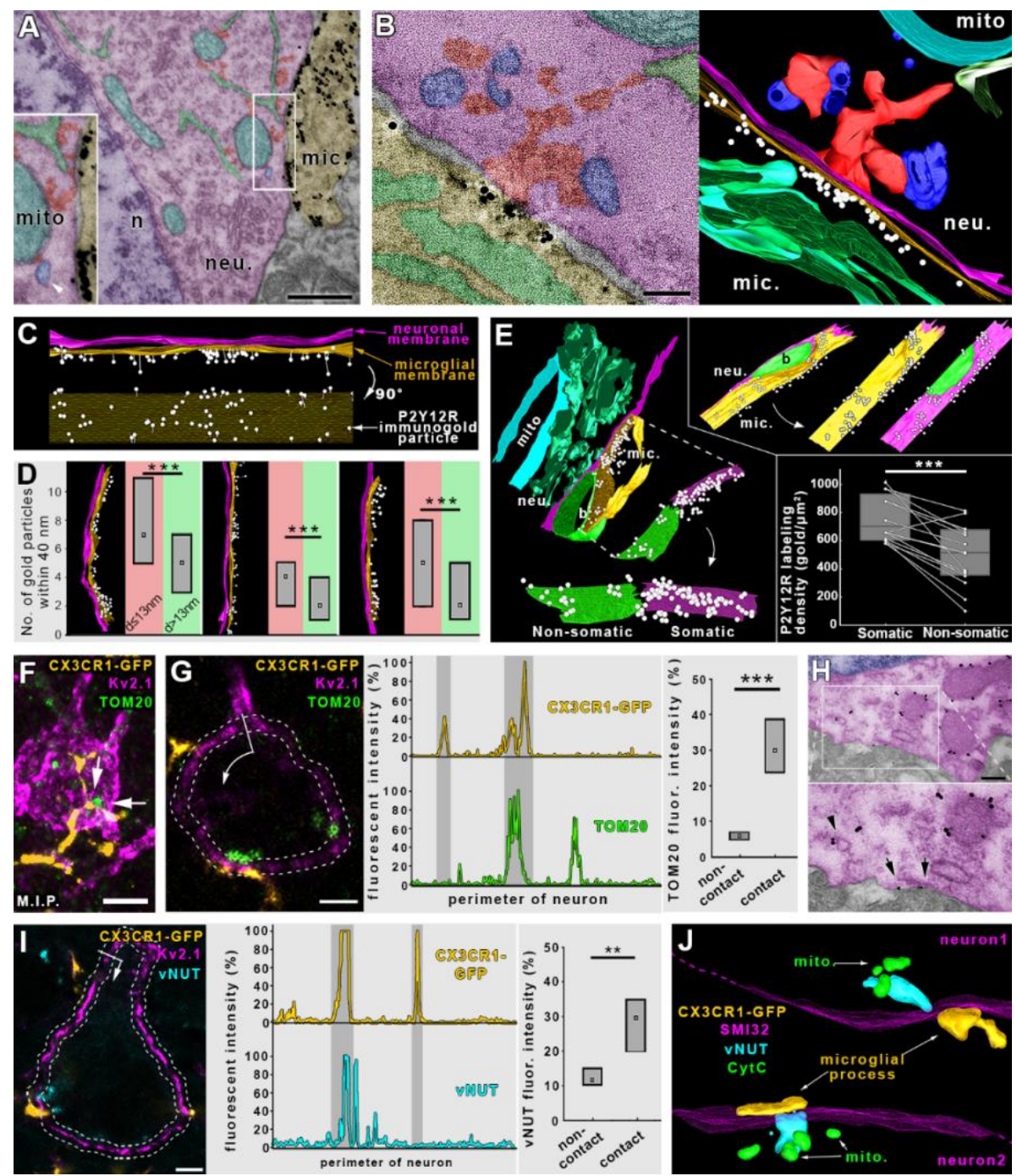

Figure 2. Microglia-neuron junctions possess a specialized nano-architecture and molecular machinery optimized

\section{for purinergic cell-to-cell communication}

(A) Transmission electron micrograph shows the area of the neuronal cell body (neu.) contacted by a P2Y12R-

immunogold (black grains) labeled microglial process (mic.). The junction possesses a unique ultrastructure with closely apposed mitochondria (mito., cyan), reticular membrane structures (green), intracellular tethers (red). A mitochondria-associated vesicle (blue, marked by white arrowhead) is also visible. The nucleus (n) of the neuron is purple. (B) $0.5 \mathrm{~nm}$ thick virtual section of an electron tomographic volume (left) and 3D model (right) shows the special nano-architecture of a somatic microglia-neuron junction (colors represent the same structures, as in panel A). Note the specific enrichment of P2Y12R labeling at the core of the junction. (C-D) P2Y12R density negatively correlates with the distance between microglial and neuronal membranes within the junctions. (E) P2Y12R density 
is highest at those surfaces of microglial processes that are in direct contact with the neuronal cell bodies (P2Y12R labeling is white, mitochondria - mito., neuron - neu., microglia - mic., bouton - b.). (F) CLSM maximal intensity projection (M.I.P.) shows microglial processes (yellow) contacting neuronal somata (magenta) with adjacent mitochondria (green). (G) Neuronal mitochondria are enriched at microglial junction sites. (H) Transmission electron micrographs show TOM20-immunogold labeling in neocortical neurons. Immunogold labeling (black grains) is specifically associated with outer mitochondrial membranes, while TOM20-positive vesicles can also be observed (arrowheads). Some immunogold particles can be found on the plasma membrane of the neurons (arrows), suggesting the exocytosis of mitochondria-derived vesicles. (I) VNUT-labeled vesicles are enriched at microglial junction sites. (J) 3D reconstruction of high-resolution confocal Z-stack shows parts of two neuronal cell bodies (magenta), both contacted by microglial processes (yellow). The vNUT signal (cyan) was concentrated between the junctions and closely positioned mitochondria (green).

For statistical details, see supplementary text for Fig. 2. 

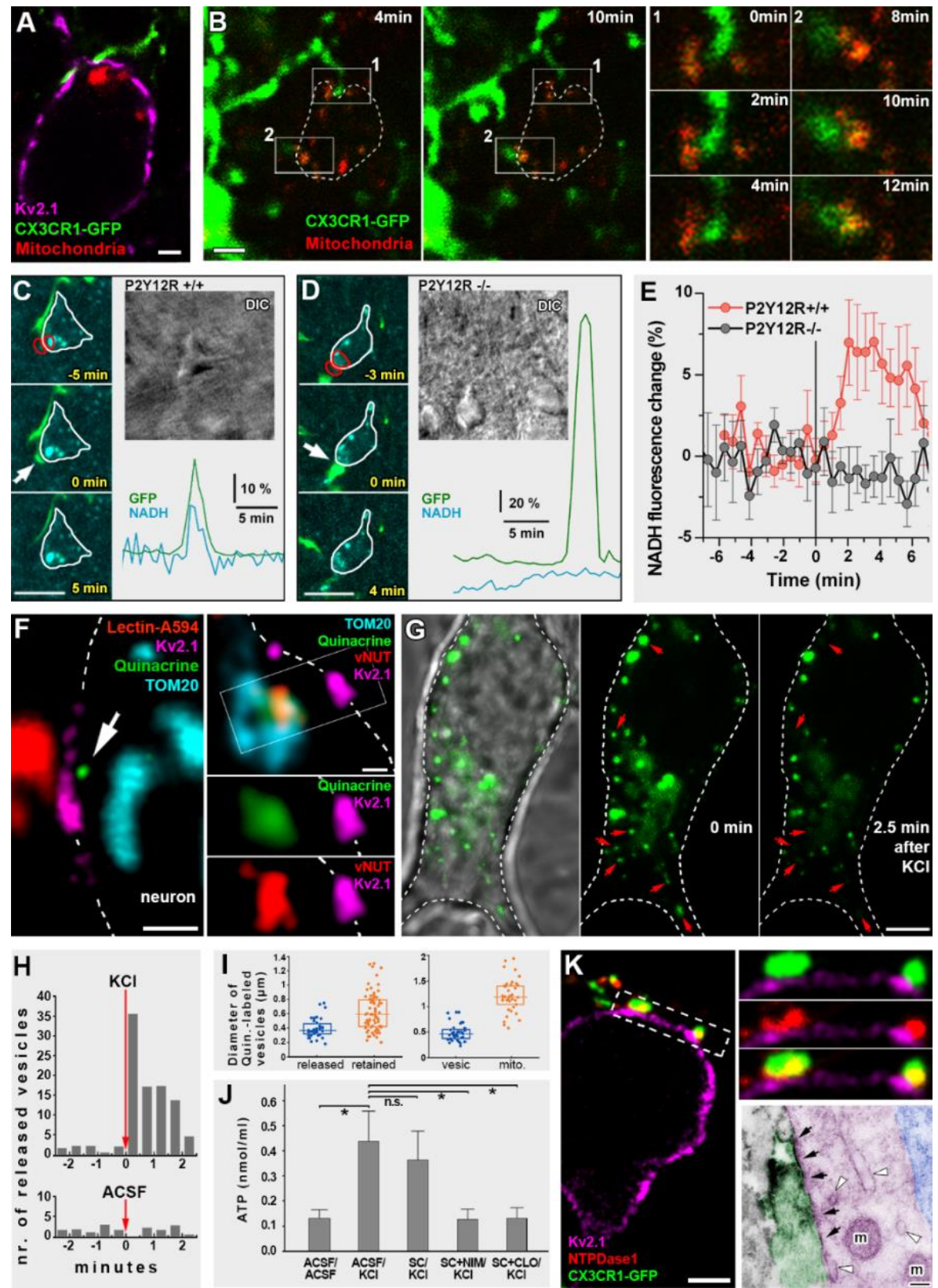

Figure 3. Neuronal mitochondrial activity and purinergic signaling is involved in microglia-neuron

\section{communication}

(A) CLSM image shows a microglial process (green) contacting Kv2.1 clusters (magenta) on a neuronal soma in the vicinity of a mitochondrion (Mito-R-Geco1, red) in perfusion-fixed brain. (B) In vivo 2P imaging of CX3CR1+/GFP mice in utero electroporated with CAG-Mito-R-Geco1 construct. Dashed line shows the outline of the neuron, green microglial processes touch neuronal cell body where somatic mitochondria are present. ROIs 1 and 2 are enlarged to show the development of somatic junctions. (C-D) Representative samples from time-lapse imaging of microglia 
show processes extending and contacting neuronal soma in CX3CR $1^{+/ G F P} / P 2 Y 12 R^{+/+}(C)$ and CX3CR1 $1^{+/ G F P} / P 2 Y 12 R^{-/-}$ (D) mice. White arrow indicates the contact site of microglia. DIC images of the imaged neurons and the fluorescence signal of GFP (green) and NADH (dark cyan) of red outlined areas are shown. (E) Average (and standard deviation) of NADH intrinsic fluorescence of all neurons in $\mathrm{P} 2 \mathrm{Y} 12 R^{+/+}($red, $n=10)$ and $\mathrm{P} 2 \mathrm{Y} 12 R^{-/-}$mice (black, $n=11$ ). (F) CLSM image shows microglial process contacting a neuronal Kv2.1 cluster with closely apposed quinacrine-labeled ATP-containing vesicle and closely localized neuronal mitochondria. Quinacrine-labeling colocalizes with vNUT-signal. (G) Images from CLSM in vitro time-lapse imaging show that quinacrine-labeled ATPcontaining vesicles (green) are released (red arrows) from neuronal cell body (white dashed outline) after KClstimulation (M.I.P. of z-stack of $2.5 \mu \mathrm{m}$ ). (H) Number of released quinacrine+ vesicles plotted as a function of time after $\mathrm{KCl}$ or vehicle treatment. (I) Size distribution of quinacrine-labeled puncta. The smaller ones (vesicles) tend to be released, and the larger ones (mitochondria) are retained. (J) $\mathrm{KCl}$ induces a robust ATP release in cultured neurons, which could not be inhibited by a mixture of the synaptic calcium-channel blockers $\omega$-Agatoxin and $\omega$ Conotoxin (SC), but is almost completely inhibited by the L-type calcium channel blocker Nimodipine (NIM), or the vNUT-inhibitor clodronate (CLO). (K) CLSM image shows robust NTPDase1 expression on microglial processes within the somatic junctions. Electron microscopic insert shows NTPDase1-labeled (dark precipitate) microglial process contacting neuronal cell body. Neuronal mitochondria $(m)$, vesicles and membrane structures (white arrowheads) are closely apposed to the contact site (black arrows), where NTPDase1 is expressed on microglial membrane. For statistical data, see supplementary text for Fig. 3. 

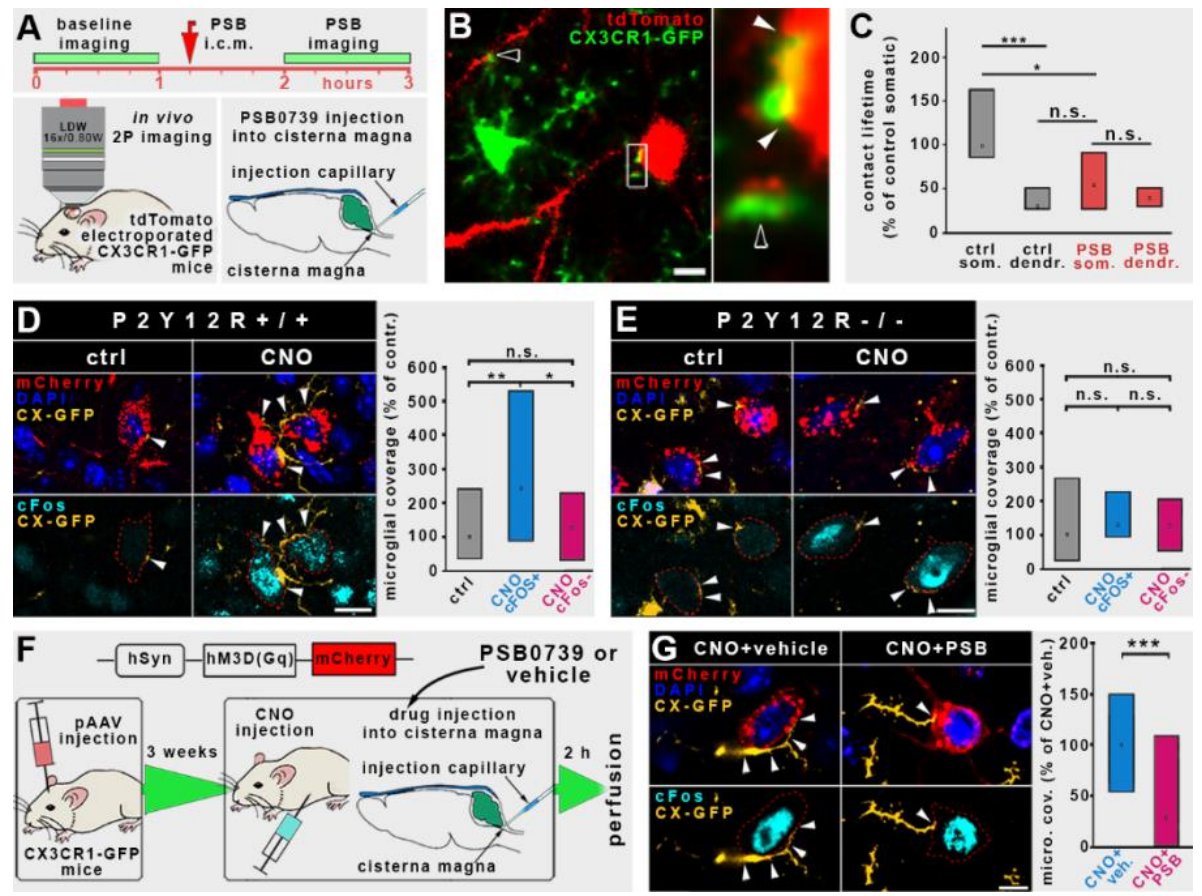

Figure 4. Physiological microglia-neuron communication at the somatic junction site is P2Y12R-dependent

(A) Outline of acute P2Y12R-blockade experiments. (B) CLSM images show examples of the recorded microglianeuron contacts. Empty arrowheads point to dendritic contacts, full arrowheads mark somatic junctions. (C) Acute i.c.m. administration of PSB significantly reduced somatic junction lifetime, but did not affect the lifetime of dendritic microglia-neuron contacts. (D) Neuronal activity induced a robust elevation of microglial process coverage of neuronal cell bodies in CNO-treated animals, but not in DREADD+/cFos-cells. (E) CNO-triggered neuronal activity could not induce an elevation of microglial process coverage of neuronal cell bodies in P2Y12R ${ }^{-}$mice. (F) Outline of combined chemogenetic - acute P2Y12R-blockade experiments. (G) Acute inhibition of microglial P2Y12Rs prevented neuronal-activity induced increase of microglial process coverage.

For statistical data, see supplementary text for Fig. 4. 

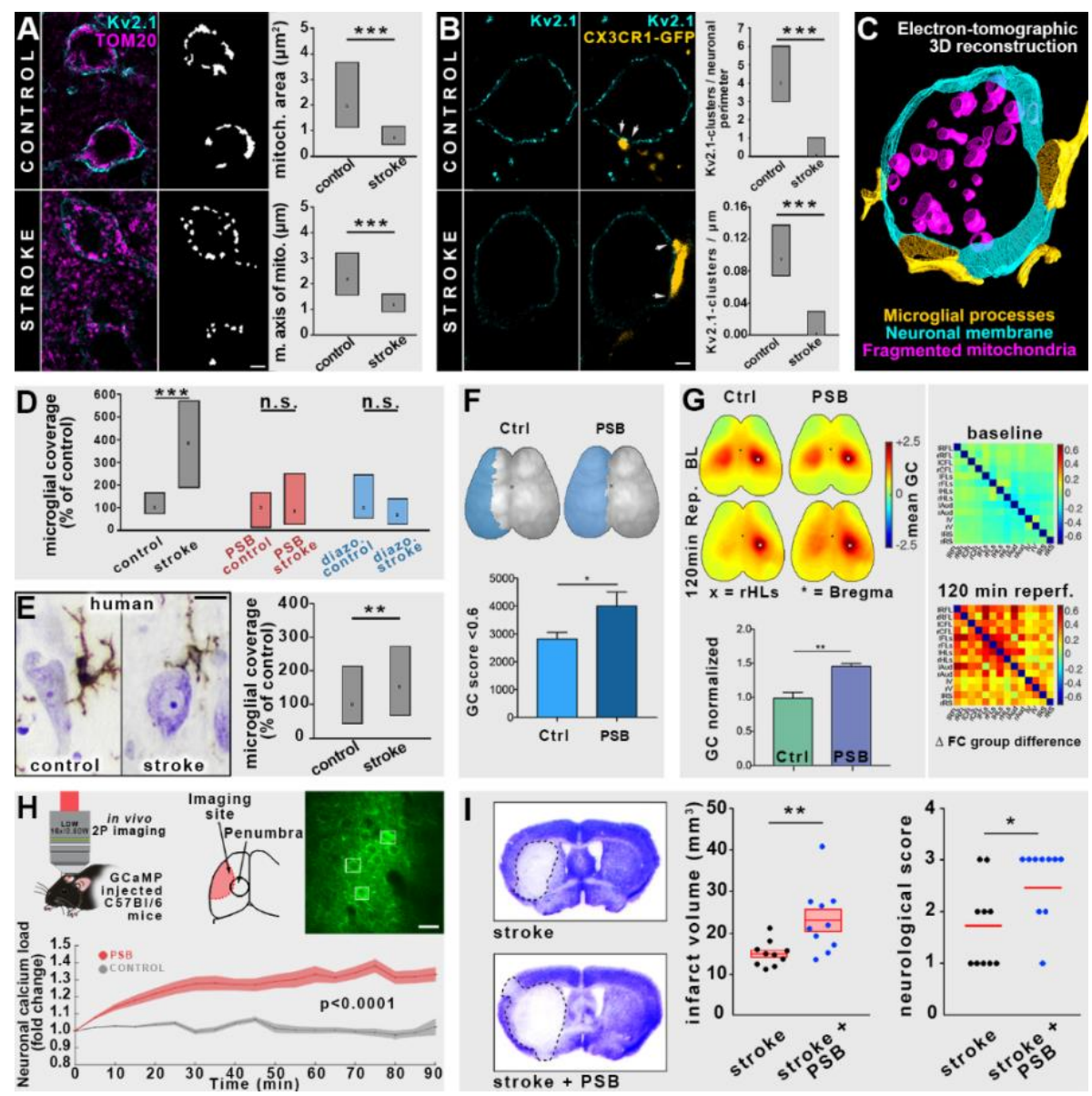

Figure 5. Microglia protect neurons after acute brain injury in a P2Y12R-dependent manner via altered somatic junctions

(A) CLSM images show that stroke induces the fragmentation of mitochondria (magenta) in neuronal cell bodies (Kv2.1 labeling, cyan) in the penumbra. Mitochondrial area and mitochondrial major axis are both significantly decreased. (B) CLSM images of cortical neurons show that in parallel with the declustering of Kv2.1-channels (cyan) microglial coverage (yellow) is significantly increased after stroke in the penumbra. (C) $3 D$ reconstruction from electron tomographic volume shows elevated microglial coverage and fragmentation of neuronal mitochondria. (D) Microglial coverage of neuronal cell bodies is robustly increased after stroke, while acute central blockade of P2Y12Rs or activation of mitochondrial ATP-sensitive potassium (KATP) channels completely abolishes the strokeinduced increase of coverage. (E) Stroke induces a 1.5-fold increase in somatic microglia coverage of human cortical neurons. (F) Topographical maps show the area of pixels with a global connectivity (GC) score less than 0.6 after ischemia. The sum of outlined pixels revealed higher dropdown of GC in PSB treated animals after stroke. (G) Left panel: Topographical maps show increased ROI-to-GC of the contralateral HLs in PSB-treated mice 120 min after 
stroke. Right panel: Seed-to-seed connectivity is increased in PSB-treated animals after stroke. (H) In vivo 2P calcium imaging reveals a significant increase of neuronal calcium load during reperfusion after acute $P 2 Y 12 R$ inhibition with PSB. (I) Infarct volume is increased after acute central P2Y12R-inhibition, which is accompanied by a significantly worse neurological outcome.

For statistical data, see supplementary text for Fig. 5. 


\title{
Science \\ MIAAAS
}

\section{Supplementary Materials for}

\author{
Microglia monitor and protect neuronal function via specialized somatic \\ purinergic junctions
}

\begin{abstract}
Csaba Cserép, Balázs Pósfai, Nikolett Lénárt, Rebeka Fekete, Zsófia I. László, Zsolt Lele, Barbara Orsolits, Gábor Molnár, Steffanie Heindl, Anett D. Schwarcz, Katinka Ujvári, Zsuzsanna Környei, Krisztina Tóth, Eszter Szabadits, Beáta Sperlágh, Mária Baranyi, László Csiba, Tibor Hortobágyi, Zsófia Maglóczky, Bernadett Martinecz, Gábor Szabó, Ferenc Erdélyi, Róbert Szipőcs, Michael M. Tamkun, Benno Gesierich, Marco Duering, István Katona, Arthur Liesz, Gábor Tamás, Ádám Dénes

Correspondence to: denes.adam@koki.mta.hu
\end{abstract}

This PDF file includes:

Materials and Methods
Supplementary Figure legends

Figures S1-S6

Tables S1-S3

Captions for Movies S1-S7

Other Supplementary Materials for this manuscript include the following:

Movies S1 to S7 


\section{Materials and Methods}

\section{Ethical considerations}

All experiments were performed in accordance with the Institutional Ethical Codex and the Hungarian Act of Animal Care and Experimentation guidelines (40/2013, II.14), which are in concert with the European Communities Council Directive of September 22, 2010 (2010/63/EU). The Animal Care and Experimentation Committee of the Institute of Experimental Medicine and the Animal Health and Food Control Station, Budapest, have also approved the experiments under the number PE/EA/1021-7/2019, $P E / E A / 673-7 / 2019$. Control human brain tissue was obtained from two female (59- and 60-years-old) and one male (73-years-old) subjects who died from causes not linked to brain diseases, and did not have a history of neurological disorders (ethical approval ETT TUKEB 31443/2011/EKU [518/PI/11]). Tissues from patients who died after ischemic stroke affecting the MCA area were obtained from two female (77- and 78-years-old) and one male (66-years-old) subjects (ethical approval ETT-TUKEB 62031/2015/EKU, 34/2016 and 31443/2011/EKU (518/PI/11)) See Supplementary Table 2. Informed consent was obtained for the use of brain tissue and for access to medical records for research purposes. Tissue was obtained and used in a manner compliant with the Declaration of Helsinki.

\section{$\underline{\text { Post-mortem human brain tissues }}$}

Brains of patients who died in non-neurological diseases were removed 3-5 $\mathrm{h}$ after death. The internal carotid and the vertebral arteries were cannulated, and the brains were perfused first with physiological saline (using a volume of $1.5 \mathrm{I}$ in $30 \mathrm{~min}$ ) containing heparin $(5 \mathrm{ml})$, followed by a fixative solution containing $4 \%$ paraformaldehyde, $0.05 \%$ glutaraldehyde and $0.2 \%$ picric acid (vol/vol) in $0.1 \mathrm{M}$ PB, pH 7.4 (4-5 I in 1.5-2 h). The cortical and hippocampal samples were removed from the brains after perfusion, and were postfixed overnight in the same fixative solution, except for glutaraldehyde, which was excluded. Blocks were dissected, and $50 \mu \mathrm{m}$ thick sections were prepared on a vibratome (VT1200S, Leica, Germany). Brains of stroke patients were removed after 10-15 h after death and immersion fixed in $4 \%$ paraformaldehyde. Small regions from the affected cerebral cortex were dissected, embedded into paraffin both from the ipsilateral and contralateral hemisphere and 6-8 $\mu \mathrm{m}$ thick sections were cut on a sledge microtome.

\section{Animals}

Experiments were carried out on 12-18 weeks old C57BL/6 (RRID:IMSR_JAX:000664), CAMK2 GFP, PVA GFP, GAD65 GFP, CX3CR1 ${ }^{+/ G F P}$ (IMSR_JAX:005582), CX3CR1 $1^{+/ G F P} / P 2 Y 12^{-/-}$and C57BL/6J-Tg(Thy1GCaMP6s)GP4.12Dkim/J mice (23, 58-61). Mice were bred and genotyped the SPF unit of the Animal Care Unit of the Institute of Experimental Medicine (IEM, Budapest, Hungary) as described earlier (37). Mice had free access to food and water and were housed under light-, humidity- and temperaturecontrolled conditions. All experimental procedures were in accordance with the guidelines set by the European Communities Council Directive (86/609 EEC) and the Hungarian Act of Animal Care and Experimentation (1998; XXVIII, section 243/1998), approved by the Animal Care and Use Committee of the IEM. All experiments were performed in accordance with ARRIVE guidelines.

$\underline{\text { In vivo pharmacological treatments and chemogenetics }}$ 
To study the effect of P2Y12 receptor (P2Y12R) antagonists in healthy mice or after experimental stroke, either a single dose of PSB0739 (Tocris, R\&D Systems, Minneapolis, USA, $15 \mu \mathrm{g}$ dissolved in saline), or vehicle was administered to the cisterna magna in $5 \mu \mathrm{l}$ final volume using a glass capillary. The diffusion of materials injected i.c.m. was controlled with i.c.m. dye injections (Fig. S5e). For experiments aiming to assess the effect of microglial P2Y12R blockade on infarct size and neurological outcome, PSB0739 was administered at reperfusion after $30 \mathrm{~min}$ MCAo. For technical reasons, mice subjected to in vivo widefield calcium imaging and two-photon imaging received i.c.m. PSB0739 60 min prior to MCAo. Brains were harvested 24 hours later for histology. Diazoxide (\#D9035, Sigma, Merck KGaA, Darmstadt, Germany) dissolved in $0.4 \%$ DMSO and $0.01 \mathrm{M} \mathrm{NaOH}$ was administered in a single dose of 10 $\mathrm{mg} / \mathrm{kg}$ intraperitoneally, immediately before reperfusion of the MCAo took place during the stroke surgeries. Brains were prepared 4 hours later for histological assessment. For selective microglia elimination, C57BL/6J mice were fed a chow diet containing the CSF1 receptor antagonist, PLX5622 (Plexxikon Inc., $1200 \mathrm{mg}$ PLX5622 in $1 \mathrm{~kg}$ chow) for 3 weeks to eliminate microglia from the brain (and another group of C57BL/6J mice were fed control chow diet). For the chemogenetic activation of neurons $0.1 \mu \mathrm{l}$ of AVV8-pAAV-hSyn-HA-hM3D(Gq)-MCherry (RRID: Addgene_50474, Addgene, USA) was injected into the neocortex of CX3CR1 ${ }^{+/ G F P}$ mice. After 3 weeks of incubation, mice received saline or clozapine-N-oxide $(0.1 \mathrm{mg} / \mathrm{ml})$ intraperitoneally to induce DREADD activation. Animals were perfused 1 hour after the injections, and processed for histology. For in vivo calcium imaging C57BL/6J mice were used. $200 \mathrm{nl}$ concentrated AAV1.Syn.GCaMP6f.WPRE.SV40 (RRID: Addgene_100837, Penn Vector Core) was injected into the cortex 200-300 $\mu \mathrm{m}$ below the surface with glass capillary. The injection coordinates were $1.5 \mathrm{~mm}$ lateral from midline, $1.2 \mathrm{~mm}$ posterior from bregma. Cranial window surgery and two-photon (2P) resonant imaging were performed 2 weeks after injection.

\section{Experimental stroke}

MCAo was performed using the intraluminal filament technique as described earlier (37). In brief, animals were anaesthetized with isoflurane and a silicone-coated monofilament $(210-230 \mu \mathrm{m}$ tip diameter, Doccol, Sharon, US) was introduced into the left external carotid artery and advanced along the internal carotid artery to occlude the MCA for 30 or 45 min. Occlusion was confirmed by a laser Doppler (Moor Instruments, UK). During surgery, core temperature was maintained at $37 \pm 0.5^{\circ} \mathrm{C}$. The following exclusion criteria were set up: animals having less than $70 \%$ relative reduction in blood flow, either having haemorrhage or having shorter survival than $24 \mathrm{~h}$ were excluded from any further analysis pre hoc. In total 2 animals (one control and one PSB treated) were excluded post hoc due to intracerebral haemorrhage, 1 PSB treated animal died before $24 \mathrm{~h}$. Altogether 3 out of 36 animals were excluded (8.33\%) from the analysis, and total mortality was $2.77 \%$.

Functional outcome of mice was assessed $24 \mathrm{~h}$ after MCAo using the corner test (62) and the 5point Bederson's sensory-motor deficit scoring system (63). Briefly, the following scores were given: a 0 , no motor deficit; 1 , flexion of torso and contralateral forelimb when mouse was lifted by the tail; 2 , circling to the contralateral side when mouse is held by the tail on a flat surface, but normal posture at rest; 3 , leaning to the contralateral side at rest, 4, no spontaneous motor activity, 5, early death due to stroke.

Infarct size was calculated based on cresyl-violet stained coronal sections as described previously (37). In brief, lesion volume at $24 \mathrm{~h}$ reperfusion was calculated by integration of areas of damage measured at eight neuro-anatomically defined coronal levels (between $2.9 \mathrm{~mm}$ rostral and $4.9 \mathrm{~mm}$ caudal to bregma) followed by correction for oedema. 
To delineate the ischemic penumbra, unfixed $1 \mathrm{~mm}$ thick brain slices were incubated in $1 \%$ TTC (2,3,5-Triphenyltetrazolium chloride, Sigma) dissolved in PBS at $37{ }^{\circ} \mathrm{C}$ for 20 minutes. Slices were then postfixed with $4 \%$ PFA in PB for 24 hours at $4{ }^{\circ} \mathrm{C}$, resectioned and processed for immunostaining.

\section{$\underline{\text { Perfusion and tissue processing for histology }}$}

Adult mice were anesthetized by intraperitoneal injection of $0.15-0.25 \mathrm{ml}$ of an anaesthetic mixture (containing $20 \mathrm{mg} / \mathrm{ml}$ ketamine, $4 \mathrm{mg} / \mathrm{ml}$ xylazine-hydrochloride). Animals were perfused transcardially with $0.9 \% \mathrm{NaCl}$ solution for 1 minute, followed by $4 \%$ freshly depolymerized paraformaldehyde (PFA) in $0.1 \mathrm{M}$ phosphate buffer (PB) pH 7.4 for 40 minutes, and finally with $0.1 \mathrm{M} \mathrm{PB}$ for 10 minutes to wash the fixative out. Blocks containing the primary somatosensory cortex and dorsal hippocampi were dissected and coronal sections were prepared on a vibratome (VT1200S, Leica, Germany) at $20 \mu \mathrm{m}$ thickness for STORM experiments, $50 \mu \mathrm{m}$ thickness for immunofluorescent experiments and electron microscopy/electron tomography.

\section{$\underline{\text { Cloning }}$}

CAG-IRES-tDTomato (pCAG-IRES-tDTomato): The GFP-polyA part of the pCAGIG plasmid (a gift from Connie Cepko, Addgene plasmid \#11159, RRID:Addgene_11159) was replaced with tDTomato-pA (a gift from Gyula Balla, IEM, Hungary) using blunt-end cloning (pCAGIG:Pstl-BstXI, pcDNA3-tDTomato: HindIIIPvull), fragments were blunted via Klenow chewback/fill-in respectively.

CAG-Mito-R-Geco: CMV-Mito-R-Geco1 (a gift from Robert Campbell; Addgene plasmid \#46021, RRID:Addgene_46021) was digested Pmel and the mito-R-Geco fragment then subcloned into EcoRVdigested pBSKII SK+ (Stratagene). Orientation was checked with restriction analysis. Then pBSKII-Mito-RGeco was cut with Acc65I-Notl and cloned into pCAG-GFP (a gift from Connie Cepko (Addgene plasmid \#11150, RRID:Addgene_11150) digested with Acc65I-NotI.

KV2.1-EGFP: CMV-hKv2.1-pEGFP-C1 was a gift from Federico Sesti (Addgene plasmid \#111538; http://n2t.net/addgene:111538; RRID:Addgene_111538)

Kv2.1-ruby: CMV-Kv2.1-Ruby2 encoding plasmid was constructed by removing the Nhel-GFP-EcoRI fragment in pEGFPC1-rKv2.1 (described earlier (53)) and replacing it with an in frame Nhel-Ruby2-EcoRI fragment.

DNKV2.1-YFP (CMV-DNKV2.1-YFP): A YFP and myc epitope tagged Kv2.1 dominant negative construct was assembled using standard PCR-based cloning methods to insert the desired nucleotide sequences. Rat Kv2.1 cDNA fragment corresponding to amino acids 1-218 and encoding the N-terminus and first transmembrane domain was amplified and tagged with the myc epitope on the $3^{\prime}$ end. This fragment was then inserted into the BamHI-Xbal sites of the pEYFP-C1 polylinker. The resulting construct expresses the Kv2.1 peptide with YFP on the $\mathrm{N}$-terminus and the myc epitope on the $\mathrm{C}$-terminus.

\section{In utero electroporation:}

Timed-pregnant C57BL/6J (Jackson) females bred with homozygous CX3CR1 ${ }^{\text {GFP/GFP }}$ transgenic animals were anesthetized by isoflurane vaporization at embryonic day 14.5. Abdominal cavity was opened longitudinally and uterine horns were exposed. Approximately $1 \mu$ of expression vector $(1 \mu \mathrm{g} / \mu \mathrm{l}$ all of the constructs) in endotoxin-free water containing Fast Green dye (Roth 1:10000 dilution) was injected into the embryonic lateral ventricles, using glass capillary and mouth pipette. Electroporation was performed with tweezer electrodes, $5 \times 50 \mathrm{~V}$ pulses of 50 millisecond duration were applied with 950 
millisecond intervals using the In Utero Electroporator SP-3c (Supertech). After the electroporation, uterine horns were returned into the abdominal cavity, the muscle wall and skin were sutured, and embryos were allowed to continue their normal development. All the littermates were CX3CR1 $1^{\text {/GFP }}$ heterozygous and were born naturally.

Mito-R-Geco1 in vitro transfection into HEK-cells

HEK-293 cells were cultured in Dulbecco's Modified Eagle Medium (4.5 g/L glucose, L-glutamine \& sodium pyruvate; Corning) with $10 \%$ heat-inactivated Fetal Bovine Serum (Biosera) and incubated at 37 ${ }^{\circ} \mathrm{C}$ in $5 \% \mathrm{CO}_{2}$ in air. On the day of transfection, cells were plated on poly-D-lysine (Sigma) coated $18 \mathrm{~mm}$ coverslips in 12-well cell culture plates. For transfection $2 \mu$ l Lipofectamine ${ }^{\circledR} 2000$ Reagent (Thermo Fisher Sc.) were mixed with $2 \mu \mathrm{g}$ Mito-R-Geco1 in Gibco $^{\circledR}$ Opti-MEM $^{\text {тM }}$ Media (Thermo Fisher Sc.) and stored in the hood approximately half an hour. Transfection solution was mixed with the culturing media and cells were incubated overnight. Next day, cells were fixed with 4\% PFA for 10 minutes, then washed with PBS. Permeabilization and blocking steps were performed by $0.1 \%$ TritonX and $5 \%$ NDS (Normal Donkey Serum; Sigma)/PBS solution for 30 mins. TOM20 antibody (1:1000) was applied in PBS for 90 mins. After several PBS washes cells were treated with secondary antibodies/PBS solution for an hour. Finally coverslips were washed in PBS and mounted with VECTASHIELD ${ }^{\circledR}$ HardSet $^{\text {TM }}$ mounting medium and sealed with nail polish. Confocal images were taken with 60X objective by Nikon A1R confocal system guide by NIS-Elements Microscope Imaging Software.

$\underline{\text { Isolation of microglial cells }}$

Primary microglial cells were isolated from astroglia/microglia mixed cell cultures, as described earlier (55). In brief, meninges were removed and tissue pieces were subjected to enzymatic dissociation, using $0.05 \% \mathrm{w} / \mathrm{v}$ trypsin and $0.05 \% \mathrm{w} / \mathrm{v}$ DNase for 10 minutes at room temperature. The cells were plated onto poly-L-lysine coated plastic dishes and were grown in Minimal Essential Medium (MEM, ThermoFisher Sc. 21090-055) supplemented with 10\% fetal bovine serum (FBS, Thermofisher 16000044), glutamine (4 mM; Sigma-Aldrich G3126), gentamycin (40 $\mathrm{g} / \mathrm{ml}$; Gentamicin Sandoz, 80 $\mathrm{mg} / \mathrm{ml}$ injection) and amphotericin B $(2.5 \mu \mathrm{g} / \mathrm{ml}$; Sigma-Aldrich A2411) in humidified air atmosphere containing $5 \% \mathrm{CO}_{2}$, at $37^{\circ} \mathrm{C}$. The culture medium was changed on the first two days and every third day afterwards. Microglial cells were isolated from 21-28 day old mixed cultures by mild trypsinization. In some experiments, microglia were isolated from P8 mice using anti-CD11b conjugated magnetic microbeads (Miltenyi Biotec 130-093-634) and magnetic-activated cell sorting (MACS), according to the manufacturer's protocol. The P8-derived cells were maintained in the presence of $10 \mathrm{nM}$ Macrophage Colony-Stimulating Factor (M-CSF; Thermofisher PMC2044) until use.

\section{$\underline{\text { HEK-microglia co-cultures and Kv2.1 construct transfections }}$}

In co-cultures used for transfection HEK293 cells were plated at $2.5 \times 10^{4} \mathrm{cell} / \mathrm{cm}^{2}$ density and microglial cells were seeded on top of HEK293 cell cultures in $2.5 \times 10^{4} \mathrm{cell} / \mathrm{cm}^{2}$ density a day prior to transfection. The cells were transfected with $1 \mu \mathrm{g}$ Kv2.1-GFP, Kv2.1-Ruby2 or DNKv2.1-YFP plasmid DNA. Transfection was carried out using Lipofectamine ${ }^{\mathrm{TM}} 3000$ Transfection Reagent (ThermoFisher Sc. L3000001) and Opti-MEM ${ }^{\text {тM }}$ Media (ThermoFisher Sc. 31985-062), according to the manufacturer's instructions. In brief, $1.5 \mu$ l Lipofectamine ${ }^{\mathrm{TM}} 3000$ reagent was mixed with $25 \mu \mathrm{l}$ Opti-MEM ${ }^{\mathrm{TM}}$ and $1 \mu \mathrm{g}$ DNA was mixed with $1 \mu \mathrm{l}$ P-3000 reagent and $25 \mu$ Opti-MEM $^{\mathrm{TM}}$. The two solutions were combined, incubated for $20 \mathrm{~min}$ at room temperature and the mix was added to the cells. Gene expression was evaluated the day after transfection. To label microglia, isolectin B4-Alexa 488 or 594 (Isolectin GS-IB4 
From Griffonia simplicifolia, Alexa Fluor ${ }^{\mathrm{TM}} 488$ or 594 Conjugate, ThermoFisher Sc. 121411 and I21413) was applied in $5 \mu \mathrm{g} / \mathrm{ml}$ during imaging sessions. Live imaging was performed in HEPES buffered ACSF ( $\mathrm{NaCl} 124.5 \mathrm{mM}, \mathrm{KCl} 2.5 \mathrm{mM}$, glucose $10 \mathrm{mM}, \mathrm{MgCl}_{2} 2 \mathrm{mM}, \mathrm{CaCl}_{2} 2 \mathrm{mM}, \mathrm{NaHCO}_{3} 8 \mathrm{mM}, \mathrm{HEPES} 20 \mathrm{mM}$ ) at room temperature on a Nikon A1R confocal microscope at 60x magnification (Plan Apo VC NA=1.2 $W D=0.31-0.28 \mathrm{~mm} F O V=215.04 \mu \mathrm{m}$ ). Images were analyzed using the NIS-Elements AR software.

\section{$\underline{\text { Neuronal cultures }}$}

Primary cultures of embryonic hippocampal cells were prepared from C57BL/6J mice on embryonic day 18 , as described earlier (55). Briefly, cells were seeded onto poly-L-lysine coated tissue culture plates or laminin coated glass coverslips at $1 \times 10^{5} \mathrm{cells} / \mathrm{cm}^{2}$ density and grown in NeuroBasal medium (ThermoFisher Sc. 21103-049) supplemented with 5\% FBS (ThermoFisher Sc. 16000044), B-27 $7^{\mathrm{TM}}$ Supplement (50x) (ThermoFisher Sc. 17504-044), GlutaMax ${ }^{\mathrm{TM}}$ Supplement (0.5 mM; ThermoFisher Sc. 35050061), gentamicin (40 $\mu \mathrm{g} / \mathrm{ml}$; Gentamicin Sandoz, $80 \mathrm{mg} / \mathrm{ml}$ injection), amphotericin B (2.5 $\mu \mathrm{g} / \mathrm{ml}$; Sigma-Aldrich A2411). Cytosine-arabinofuranoside (CAR, $10 \mu \mathrm{M}$; Sigma-Aldrich C1768) was added to the cultures 24-120 h after plating to limit glia growth. $48 \mathrm{~h}$ after CAR treatment half of the culture medium was changed to BrainPhys-SM1 medium (BrainPhys ${ }^{\mathrm{TM}}$ Neuronal Medium and SM1 Kit, Stemcell Technologies 05792) without FBS. Medium change was repeated every 3-4 days thereafter. Media of cultures treated with CAR as early as DIV1 were supplemented with astroglial conditioned media (1:1) collected from 1-2 week old primary astrocytic cultures after $72 \mathrm{~h}$ incubation. Primary neuronal cultures were cultivated for $7-14$ days at $37^{\circ} \mathrm{C}$ in $5 \% \mathrm{CO}_{2}, 95 \%$ air atmosphere.

\section{$\underline{\text { In vitro quinacrine experiments }}$}

To monitor vesicular ATP release, neuronal cultures were incubated with $20 \mu \mathrm{M}$ quinacrinedihydrochloride (Sigma-Aldrich Q3251) for $20 \mathrm{~min}$ at $37{ }^{\circ} \mathrm{C}$. Live imaging was performed in HEPES buffered ACSF ( $\mathrm{NaCl} 124.5 \mathrm{mM}, \mathrm{KCl} 2.5 \mathrm{mM}$, glucose $10 \mathrm{mM}, \mathrm{MgCl}_{2} 2 \mathrm{mM}, \mathrm{CaCl}_{2} 2 \mathrm{mM}, \mathrm{NaHCO}_{3} 8 \mathrm{mM}$, HEPES $20 \mathrm{mM}$ ) at room temperature on a Nikon A1R confocal microscope at 60x magnification (Plan Apo VC NA=1.2 WD=0.31-0.28mm FOV=215.04 $\mu \mathrm{m}$ ). Images were analyzed using the NIS-Elements AR software. For neuron-microglia cocultures microglial cells were seeded on top of primary neuronal cultures in $2.5 \times 104$ cell $/ \mathrm{cm}^{2}$ density a day prior to quinacrine-loading and imaging.

\section{Quantification of ATP}

The levels of ATP was determined in culture media by using HPLC method. The primary cultures were highly enriched in neurons, as non-neuronal cell proliferation was blocked by CAR as early as DIV1, as described above. Cell culture supernatants were collected at DIV7, $5 \mathrm{~min}$ after $40 \mathrm{mM} \mathrm{KCl}$ treatment, preceded by a 20 min incubation period with combinations of the following drugs: Nimodipine ( $20 \mu \mathrm{M}$; Alomone Labs N-150), Clodronate disodium (10 $\mathrm{M}$; Sigma Aldrich D4434), Omega-Agatoxin IVA (100 $\mathrm{nM}$; Alomone Labs STA-500), Omega-Conotoxin GVIA (1 $\mu \mathrm{M}$; Alomone Labs SNX-124). Media (400 $\mu \mathrm{l} /$ well) were separated into a cold Eppendorf tube which contained $50 \mu \mathrm{l}$ of homogenization solution (0.1 M perchloric acid containing theophylline as an internal standard at $10 \mu \mathrm{M}$ concentration). Perchloric anion from the supernatant was precipitated by $1 \mathrm{M}$ potassium hydroxide, the precipitate was then removed by centrifugation. The extracted purines were kept at $-20{ }^{\circ} \mathrm{C}$ until analysis. The adenine nucleotides and adenosine in culture media were determined by online column switching separation using Discovery HS C18 $50 \times 2 \mathrm{~mm}$ and $150 \times 2 \mathrm{~mm}$ columns. The flow rate of the mobile phases ["A" $10 \mathrm{mM}$ potassium phosphate, $0.25 \mathrm{mM}$ EDTA "B" with $0.45 \mathrm{mM}$ octane sulphonyl acid sodium salt, $8 \%$ acetonitrile (v/v), $2 \%$ methanol (v/v), pH 5.2] was 350 or $450 \mu \mathrm{l} / \mathrm{min}$, respectively, in a 
step gradient application. The enrichment and stripping flow rate of buffer [10 $\mathrm{mM}$ potassium phosphate, pH 5.2] was during $4 \mathrm{~min}$ and the total runtime was $55 \mathrm{~min}$. The HPLC system used was a Shimadzu LC-20 AD Analytical \& Measuring Instruments System, with an Agilent 1100 Series Variable Wavelength Detector set at $253 \mathrm{~nm}$. Concentrations were calculated by a two-point calibration curve using internal standard method.

\section{Platelet aggregation in response to P2Y12 receptor inhibition after stroke}

12-14 weeks old male C57BL/6J mice were subjected to 30 min MCA occlusion and immediately after the induction of reperfusion a specific P2Y12 receptor inhibitor (PSB0739, \#3983 Tocris, $15 \mu \mathrm{g}$ in 5 $\mu \mathrm{l}$ volume) or vehicle (PBS) was administered to the cisterna magna. $1 \mathrm{~h}$ later mice were sacrificed and venipuncture was performed from the vena cava inferior. Platelet-rich plasma samples from anticoagulated ( $3.8 \%$ Sodium citrate) blood samples were isolated by centrifugation ( $100 \times \mathrm{g}, 10 \mathrm{~min})$, were plated $\left(30 \mu \mathrm{l} /\right.$ well in a 24-well plate) and incubated at $37{ }^{\circ} \mathrm{C}$ for $30 \mathrm{~min}$ before treatment with 0.5 $\mathrm{mM}$ ADP for $1 \mathrm{~h}$ at $37^{\circ} \mathrm{C}$. ADP-induced platelet activation was measured by FACS based on CD62-P and CD42d fluorescence intensity, as described earlier in $(40,64)$. After 10 min antibody staining (CD62-PAPC, 1:400, \#17-0626-80 eBioScience, CD42d-PE, 1:400, \#12-0421-80 eBioScience) samples were lysed to remove any red blood cell contamination (BD FACS Lysing Solution), acquired with a BD FACSVerse instrument, and analyzed with BD FACSSuite software (BD Biosciences). Changes in CD62P mean fluorescence intensity values were determined on CD42d-positive platelets.

$\underline{\text { In vivo two-photon imaging }}$

Animals were anaesthetized using fentanyl (100-200 $\mu \mathrm{l}$ ). Cranial window (3 mm diameter) was opened on the left hemisphere above the primary somatosensory area and supplementary somatosensory area border (centered $3 \mathrm{~mm}$ lateral and $2 \mathrm{~mm}$ posterior to bregma) without hurting the dura mater. After removal of the skull bone a $3 \mathrm{~mm}$ and $5 \mathrm{~mm}$ double glass coverslip construct was fixed with $3 \mathrm{M}^{\mathrm{TM}}$ Vetbond ${ }^{\mathrm{TM}}$ tissue glue on top of the dura mater. Then a custom made metal headpiece (Femtonics Ltd., Budapest, Hungary) was fixed with dental cement on the surface of the skull. All experiments were performed on a Femto2D-DualScanhead microscope (Femtonics Ltd., Budapest, Hungary) coupled with a Chameleon Discovery laser (Coherent, Santa Clara, USA). For tDTomato electroporated animals the wavelength of the laser was set to $920 \mathrm{~nm}$ to measure the tdTomato and GFP signals simultaneously. For Mito-R-Geco1 mitochondrial electroporated animals the wavelength was set to $1000 \mathrm{~nm}$. Following excitation the fluorescent signal was collected using a Nikon 18X water immersion objective. Data acquisition was performed by MES software (Femtonics Ltd.). Since it has recently been shown that volatile anesthetics such as isoflurane may influence microglial process motility (54), we used fentanyl anaesthesia for these studies, which did not block microglial responses. (Fig. S5b; median process motility observed: isoflurane $0.6 \mu \mathrm{m} / \mathrm{min}, 0.3-0.83$ interquartile; fentanyl 0.6 $\mu \mathrm{m} / \mathrm{min}, 0.42-0.78$; urethane $0.48 \mu \mathrm{m} / \mathrm{min}, 0.36-0.84 ; \mathrm{n}=153$ processes from 9 animals).

To analyze contacts established by microglial processes on neuronal cell bodies and proximal dendrites, we used in utero tdTomato electroporated CX3CR1 ${ }^{+/ G F P}$ mice. To visualize microglial processes and neuronal mitochondria simultaneously, we used Mito-R-Geco1 electroporated CX3CR1 ${ }^{+/ G F P}$ mice. Galvano Z-stacks of 7 images (820x820 pixels, $5 \mu \mathrm{m}$ step size, range $=200-225 \mu \mathrm{m}$ from pial surface) were made at every 2 or 2.5 minutes. Two-photon image sequences were exported from MES and analyzed using FIJI. Dual colour images were analyzed with the Manual Tracking plugin of FIJI. We applied a local maximum centering correction method with a search square of 5 pixels. Pixel size was $167 \mathrm{~nm} / \mathrm{px}$. 
Microglial process velocity was measured on time-series images acquired with 2P microscopy. Following motion correction, monocolour images from the same region of CX3CR1 ${ }^{+/ G F P}$ mice taken 135 seconds apart were analyzed with the Manual Tracking plugin of FIJI. We applied a local maximum centring correction method with a search square of 5 pixels. Pixel size was $167 \mathrm{~nm} / \mathrm{px}$. Processes were included in the measurement, when they were clearly traceable for at least 10 minutes. The GCaMP6f signal was imaged with the laser wavelength set to $920 \mathrm{~nm}$, using the resonant scanner at $32.75 \mathrm{~Hz}$. Image size was $512 \times 488$ pixels.

$\underline{\text { Immunofluorescent labeling and confocal laser scanning microscopy (CLSM) }}$

Before the immunofluorescent staining, the $50 \mu \mathrm{m}$ thick sections were washed in PB and Trisbuffered saline (TBS). This was followed by blocking for 1 hour in 1\% human serum albumin (HSA; SigmaAldrich) and $0.1 \%$ Triton $\mathrm{X}-100$ dissolved in TBS. After this, sections were incubated in mixtures of primary antibodies overnight at room temperature. After incubation, sections were washed in TBS and were incubated overnight at $4{ }^{\circ} \mathrm{C}$ in the mixture of secondary antibodies, all diluted in TBS. Secondary antibody incubation was followed by washes in TBS, PB, the sections were mounted on glass slides, and coverslipped with Aqua-Poly/Mount (Polysciences). Immunofluorescence was analyzed using a Nikon Eclipse Ti-E inverted microscope (Nikon Instruments Europe B.V., Amsterdam, The Netherlands), with a CFI Plan Apochromat VC 60X oil immersion objective (numerical aperture: 1.4) and an A1R laser confocal system. We used 405, 488, 561 and $647 \mathrm{~nm}$ lasers (CVI Melles Griot), and scanning was done in line serial mode, pixel size was $50 \times 50 \mathrm{~nm}$. Image stacks were obtained with NIS-Elements AR software and deconvolved using Huygens Professional software (www.svi.nl). For primary and secondary antibodies used in this study, please see Supplementary Table 3.

\section{Quantitative analysis of CLSM data}

Quantitative analysis of each dataset was performed by at least two observers, who were blinded to the origin of the samples, the experiments and did not know of each other's results. For the analysis of somatic junction prevalence, confocal stacks with double immunofluorescent labeling (cell typemarker and microglia) were acquired from at least three different regions of mouse cortex. All labeled and identified cells were counted, when the whole cell body was located within the Z-stack. Given somata were considered to be contacted by microglia, when a microglial process clearly touched it (i.e. there was no space between neuronal soma and microglial process) on at least $0.5 \mu \mathrm{m}$ long segment.

For the analysis of synaptic contact prevalence, confocal stacks with triple immunofluorescent labeling (pre- and postsynaptic markers and microglia) were analyzed using an unbiased, semi-automatic method. First, the two channels representing the pre- and postsynaptic markers were exported from a single image plane. The channels were thresholded automatically in FIJl, the "fill in holes" and "erode" binary processes applied. After automatic particle tracking, synapses were identified where presynaptic puncta touched postsynaptic ones. From these identified points we selected 200/animal in a systematic random manner. After this, the corresponding synapses were found again in the original Z-stacks. A synapse was considered to be contacted by microglia, when a microglial process was closer than $200 \mathrm{~nm}$ (4 pixels on the images).

To measure the distribution of Kv2.1 and Kv2.1 labeling relative to microglial processes, confocal stacks were exported into single-channel TIFF-series. Identical measuring frames $\left(1.32 \mu \mathrm{m}^{2}\right)$ were placed randomly along the surface of pyramidal cells and integrated Kv2.1 fluorescent density was measured in 
each frame in FIJI. Afterwards, frames containing microglial contacts were identified ("contact" group) and compared with frames not containing microglial processes ("non-contact" group).

For the measurements of mitochondrial fragmentation, we used tissue from mice that were sacrificed 4 hours after a one hour-long unilateral MCAo. Confocal stacks with double immunofluorescent labeling (Kv2.1 and TOM20) were taken from the penumbra and the corresponding contralateral region. We used the Kv2.1 labeling to trace neuronal cell bodies as regions of interest (ROI). Every cell was counted once using the confocal plane containing its largest cross-section. Within the ROIs TOM20 labeling was investigated with FIJI: after automatic thresholding, we ran the 'Analyze Particles' command to obtain the area and the major axis of individual somatic mitochondria.

For the analysis of Kv2.1 clusters, individual cells were measured by using the confocal Z-plane containing the largest cross-section of the cell body. The intensity profile of Kv2.1 labeling was plotted using FIJI. A cluster was identified when at least three adjacent pixels' intensity was more than 25 grayscale values (10\% of an 8 -bit image) larger than the average fluorescent intensity of that particular cells Kv2.1 labeling.

Microglial process coverage was measured on CLSM Z-stacks acquired with a step size of $300 \mathrm{~nm}$. On single-channel images, Kv2.1-positive cells were selected randomly, the cell bodies of which were fully included in the captured volume. The surface of these cells was calculated by measuring the circumference of the soma on every section multiplied by section thickness. The length of microglial process contacts was measured likewise.

TOM20 and vesicular nucleotide transporter (vNUT) fluorescent intensity profiles were analyzed using a semi-automatic method. Confocal stacks with triple immunofluorescent labeling (microglia, Kv2.1 and TOM20/vNUT) were collected. The section containing the largest cross-section of a pyramidal cell was used to trace the cell membrane according to Kv2.1-labeling. This contour was then expanded and narrowed by $0.5 \mu \mathrm{m}$ to get an extracellular and an intracellular line, respectively. The intensity of fluorescent labeling was analyzed along these lines. After normalizing and scaling, microglial contact was identified where microglial fluorescent intensity was over $20 \%$ of total, for at least $500 \mathrm{~nm}$. Then the contact area was extended 500-500 nm on both sides, and TOM20/vNUT fluorescent intensity within these areas was measured for "contact" value. 3-dimensional reconstruction of CLSM and 2P imaging stacks was performed using the IMOD software package (65).

\section{STORM super-resolution imaging}

Free-floating brain sections were blocked with $2 \%$ normal donkey serum followed by immunostaining with rabbit anti-P2Y12R and mouse anti-Kv2.1 antibodies, followed by anti-rabbit Alexa 647 and anti-mouse Alexa 594 secondary antibodies. Sections were mounted onto \#1.5 borosilicate coverslips and covered with imaging medium containing 5\% glucose, $0.1 \mathrm{M}$ mercaptoethylamine, 1 $\mathrm{mg} / \mathrm{ml}$ glucose oxidase, and catalase (Sigma, $1500 \mathrm{U} / \mathrm{ml}$ ) in Dulbecco's PBS (Sigma), immediately before imaging. STORM imaging was performed for P2Y12R (stimulated by a $647 \mathrm{~nm}$ laser) by using a Nikon NSTORM C2+ superresolution system that combines 'Stochastic Optical Reconstruction Microscopy' technology and Nikon's Eclipse Ti research inverted microscope to reach a lateral resolution of $20 \mathrm{~nm}$ and axial resolution of $50 \mathrm{~nm}$ (26). Imaging was performed using the NIS-Elements AR 4.3 with N-STORM 3.4 software, and we used VividSTORM open-source software. Molecule lists were exported from NIS in txt format, and the three image planes of the ics-ids file pairs from the deconvolved confocal stacks matching the STORM volume were converted to the ome-tiff format using FIJI software. Confocal and 
corresponding STORM images were fitted in VividSTORM. Localization points exceeding a photon count of 2000 were counted as specific superresolution localization points. Local density filter (10 neighbours within $150 \mathrm{~nm}$ for P2Y12R and Iba1, and 5 neighbours within $150 \mathrm{~nm}$ for Kv2.1) and Z-filter ( $\pm 300 \mathrm{~nm}$ from focal plane) was applied to the localization points.

\section{Pre-embedding immunoelectron microscopy}

After extensive washes in PB and $0.05 \mathrm{M}$ TBS sections were blocked in 1\% HSA in TBS. Then, they were incubated in primary antibodies (Supplementary Table 3) diluted in TBS containing $0.05 \%$ sodium azide for 2-3 days. After repeated washes in TBS, the sections were incubated in blocking solution (GelBS) containing $0.2 \%$ cold water fish skin gelatine and $0.5 \%$ HSA in TBS for $1 \mathrm{~h}$. Next, sections were incubated in gold-conjugated or biotinylated secondary antibodies (Supplementary Table 3) diluted in Gel-BS overnight. After extensive washes in TBS the sections were treated with $2 \%$ glutaraldehyde in 0.1 M PB for 15 min to fix the gold particles into the tissue. This was occasionally followed by incubation in avidin-biotinylated horseradish peroxidase complex (Elite ABC; 1:300; Vector Laboratories) diluted in TBS for $3 \mathrm{~h}$ at room temperature or overnight at $4{ }^{\circ} \mathrm{C}$. The immunoperoxidase reaction was developed using 3,3-diaminobenzidine (DAB; Sigma-Aldrich) as chromogen. To enlarge immunogold particles, sections were incubated in silver enhancement solution (SE-EM; Aurion) for 40-60 min at room temperature. The sections were then treated with $1 \%$ (for electron tomography) or $0.5 \% \mathrm{OsO}_{4}$ in $0.1 \mathrm{M}$ $\mathrm{PB}$, at room temperature (for electron tomography) or on ice, dehydrated in ascending alcohol series and in acetonitrile and embedded in Durcupan (ACM; Fluka). During dehydration, the sections were treated with $1 \%$ uranyl acetate in $70 \%$ ethanol for $20 \mathrm{~min}$. For electron microscopic analysis, tissue samples from the CA1 area of dorsal hippocampus/somatosensory cortex (S1) were glued onto Durcupan blocks. Consecutive $70 \mathrm{~nm}$ thick (for conventional electron microscopic analysis) or $150 \mathrm{~nm}$ thick (for electron tomography) sections were cut using an ultramicrotome (Leica EM UC6) and picked up on Formvar-coated single-slot grids. Ultrathin sections for conventional electron microscopic analysis were examined in a Hitachi 7100 electron microscope equipped with a Veleta CCD camera (Olympus Soft Imaging Solutions, Germany). $150 \mathrm{~nm}$ thick electron tomography sections were examined in FEI Tecnai Spirit G2 BioTwin TEM equipped with an Eagle 4k HS camera.

\section{Electron tomography}

For the electron tomographic investigation, we used $150 \mathrm{~nm}$ thick sections from the hippocampal CA1 region from the anti-P2Y12R immunogold stained material (see: "Pre-embedding immunoelectronmicroscopy"). Before electron tomography, serial sections on single-slot copper grids were photographed with a Hitachi H-7100 electron microscope and a Veleta CCD camera. After this, grids were put on drops of $10 \%$ HSA in TBS for 10 minutes, dipped in distilled water (DW), put on drops of 10 $\mathrm{nm}$ gold conjugated Protein-A (Cytodiagnostics \#AC-10-05) in DW (1:3), and washed in DW. Finally, we deposited $5 \mathrm{~nm}$ thick layers of carbon on both sides of the grids. Electron tomography was performed using a Tecnai T12 BioTwin electron microscope equipped with a computer-controlled precision stage (CompuStage, FEI). Acquisition was controlled via the Xplore3D software (FEI). Regions of interest were pre-illuminated for 4-6 minutes to prevent further shrinkage. Dual-axis tilt series were collected at 2 degree increment steps between -65 and +65 degrees at $120 \mathrm{kV}$ acceleration voltage and 23000x magnification with $-1.6--2 \mu \mathrm{m}$ objective lens defocus. Reconstruction was performed using the IMOD software package (65). Isotropic voxel size was $0.49 \mathrm{~nm}$ in the reconstructed volumes. After combining the reconstructed tomograms from the two axes, the nonlinear anisotropic diffusion filtering algorithm 
was applied to the volumes. Segmentation of different profiles has been performed on the virtual sections using the 3Dmod software, and measurements were done on the scaled 3D models.

Analysis of the connection between membrane distance and P2Y12R density was carried out by investigating all points of the microglial membrane facing neuronal soma, using reconstructed 3D models. Coordinates of the points of neuronal membrane, soma-facing microglial membrane and P2Y12R labeling gold particles were exported using IMOD. For every single point of microglial membrane, the lowest distance to the neuronal membrane and the number of P2Y12R labeling gold particles within $40 \mathrm{~nm}$ was calculated with a unique algorithm running in program $R$ (The R Foundation). Since neuronal junctions established by microglial processes are dynamic, a strong linear correlation cannot be expected; therefore statistical analysis was carried out by dividing data into two groups. In the analyzed tomograms the average distance between neuronal somatic membranes and facing microglial membranes was $13.06 \mathrm{~nm}$, which we used as demarcation point.

Analysis of P2Y12R density along different surfaces of microglial processes was done using reconstructed 3D models. We identified segments of microglial membranes facing (running parallel with) different neuronal membranes. These segments were grouped depending on opposing neuronal profiles (e.g. neuronal soma or other neuronal parts). Using IMOD, the surfaces of microglial profiles were measured and gold particles were assigned to the closest membrane part. Due to different labeling density and penetration differences, we only performed pairwise comparisons between 'somatic' and 'non-somatic' microglial membranes within the same microglial processes. Only those gold particles were counted that localized within $40 \mathrm{~nm}$ of the microglial membrane.

$\underline{\text { In vitro nicotinamide adenine dinucleotide (NADH) imaging }}$

Mice were anaesthetized by inhalation of halothane, and following decapitation $200 \mu \mathrm{m}$ thick coronal slices were prepared from the somatosensory and visual cortex with a vibrating blade microtome (Microm HM $650 \mathrm{~V}$ ) immersed in slicing solution containing (in $\mathrm{mM}$ ): $130 \mathrm{NaCl}, 3.5 \mathrm{KCl}, 1$ $\mathrm{NaH}_{2} \mathrm{PO}_{4}, 24 \mathrm{NaHCO}_{3}, 1 \mathrm{CaCl}_{2}, 3 \mathrm{MgSO}_{4}, 10 \mathrm{D}(+)$-glucose, saturated with $95 \% \mathrm{O}_{2}$ and $5 \% \mathrm{CO}_{2}$. The solution used during experiments was identical to the slicing solution, except it contained $3 \mathrm{mM} \mathrm{CaCl}_{2}$ and $1.5 \mathrm{mM} \mathrm{MgSO}_{4}$. Experiments were carried out less than 4 hours after slicing. During image acquisition slices were kept at $\sim 36{ }^{\circ} \mathrm{C}$. Imaging with multiphoton excitation was performed using a Zeiss LSM 7MP scanning microscope (Carl Zeiss, Germany) through a 40X water-immersion objective (W-Plan, NA 1.0, Carl Zeiss). To acquire simultaneous excitation of GFP and NADH autofluorescence of acute brain slices we used two single wavelength mode-locked Ti:sapphire lasers the beams of which were coupled to each other by a dichroic beam splitter (t810lpxr, Chroma Technology Corp, USA). One of our lasers (MaiTai DeepSee, Spectra-Physics, Santa Clara, USA) exciting GFP operated at $885 \mathrm{~nm}$, while our second laser (FemtoRose 100 TUN, R\&D Ultrafast Lasers, Hungary) exciting NADH had an operation wavelength of $750 \mathrm{~nm}$. Both laser systems delivered $\sim 100 \mathrm{fs}$ pulses at $\sim 80 \mathrm{MHz}$ and $\sim 76 \mathrm{MHz}$ repetition rate, respectively. A total average laser power of $16-18 \mathrm{~mW}$ was measured after the objective during imaging. Time-lapse images (1024x1024 pixels) were collected continuously for up to 55 minutes with 30.98 seconds frame scan time. Emission filters were chosen to separate intrinsic NADH fluorescence (ET460/50m, Chroma Technology Corp, USA) from GFP fluorescence (ET525/50m, Chroma Technology Corp, USA). Time lapse images were processed and analyzed in FIJI (ImageJ, NIH) software. As a first step images were spatial filtered (mean filter smooth with 1 pixel diameter) and corrected for contrast. To remove jitter in image series stabilization was applied with a FIJI plugin (K. Li, "The image stabilizer plugin for ImageJ," http://www.cs.cmu.edu/ kangli/code/Image_Stabilizer.html, February, 2008). 
Analysis and quantification of NADH fluorescence was carried out in manually selected areas of compartments of cell bodies at microglial contact site.

\section{In vivo widefield calcium imaging}

In vivo widefield calcium imaging was performed as previously described in detail (66). In brief, as an optogenetic calcium-reporter mouse strain, C57BL/6J-Tg(Thy1-GCaMP6s)GP4.12Dkim/J (61) heterozygous mice were bred at the Institute for Stroke and Dementia Research, Munich. The skin covering the skull and the underlying connective tissue were removed in head-fixed mice and a layer of transparent dental cement was distributed on the window area and covered with a coverslip. Afterwards, the mice were allowed to recover from the surgery for more than $48 \mathrm{~h}$ before the first image acquisition. For image acquisition, mice were injected with $0.05 \mathrm{mg} / \mathrm{kg}$ bodyweight of medetomidine intraperitoneally 5 minutes prior to inducing inhalation anesthesia with a mixture of $5 \%$ isoflurane in $70 \%$ nitrous oxide and $30 \%$ oxygen. After 70 seconds, the animals were fixed in a stereotactic frame, the dose of isoflurane was decreased to $1.5 \%$ for 140 seconds and finally decreased to $0.75 \%$ for 4 minutes to maintain steady-state before data-acquisition. In vivo widefield calcium imaging was performed on a custom-built imaging setup described in (66). This setup allowed widefield imaging through the chronic window on top of the skull into the cortex of both forebrain hemispheres by covering a field-of-view of $12 \times 12 \mathrm{~mm}$, corresponding to an image matrix of 330x330 pixels. Image acquisition was conducted for 29 minutes (44 x 1000 frames, immediately after MCAo induction, starting $60 \mathrm{~min}$ after injection of PSB) or 4 minutes ( $6 \times 1000$ frames, baseline acquisition, starting 60 min after injection of PSB and after 120 min reperfusion, respectively). After the imaging session, anesthesia was terminated by intraperitoneally injecting the mice with $0.1 \mathrm{mg} / \mathrm{kg}$ bodyweight Atipamezole. During all anesthetized procedures body temperature was maintained using a feedbackcontrolled heating system. After end of surgeries animals were put in a heating chamber until they had recovered from anesthesia. Post-surgery analgesia and sedation protocols were conducted in accordance with approved protocols by the governmental committee.

Images of every acquisition were masked in a two-step procedure. First, a general mask was applied to exclude lateral cortical areas, which were out of focus due to the curvature of the cortical surface as described in (66). Second, an individual mask was computed to exclude all pixels, in which the calcium signal was saturated due to autofluorescence, as occurring in areas affected by the infarct (66). Both, the general and the individual masks were combined for every acquisition.

To characterize changes in the cortical network after stroke, functional connectivity was computed between pairs of ROls, representing functional cortical areas, previously defined (66). Functional connectivity was calculated as the Fisher z-transformed Pearson-moment correlation between the ROI signal time-courses. The average connectivity scores were calculated within each group (PSB-treated and control group), and the difference between groups was depicted for all ROI pairs in a heatmap.

For seed-based functional connectivity analysis, connectivity scores were calculated in the same way but between a selected ROI in the right hindlimb sensory area ( $\mathrm{rHLs}$ ) and the signal time series of all pixels on the cortical surface included in the combined general and individual masks. In order to quantify connectivity change of the rHLs after stroke, the connectivity scores were normalized by dividing through the connectivity scores resulting in the baseline condition. Results were visualized as topographical maps of all brain pixel. 
Overall functional connectivity alterations due to treatment of the mice with PSB (naïve and stroke) were assessed by computing the global connectivity (GC) for each pixel inside the combined general and individual mask. For a given pixel, GC was calculated by calculating the functional connectivity with each other pixel inside the mask, followed by averaging across the resulting connectivity scores. In order to assess treatment effects, GC scores were averaged pixel-wise within group (PSB and control group). The difference between groups was then visualized as topographical map of all brain pixels.

To compare the extent of GC dropdown between PSB and control treated animals after stroke, the sum of all pixels with a moderate GC (i.e. global connectivity less than 0.6 according to (67)) was calculated for every mouse. The mean area of each group was contoured within the GC map and the area was assessed quantitatively per animal and compared between the groups.

To represent the non-functionality of cortical tissue during the occlusion of the middle cerebral artery, the number of saturated pixels during the occlusion was calculated. During the occlusion, cortical spreading depressions (CSDs) appeared. Given the high degree of neuronal activation within areas covered by these waves, the high amplitude of the $\mathrm{Ca}^{2+}$ derived signal caused saturation in these areas. Therefore, we quantified the spatial extent of CSDs by counting the saturated pixels during these waves. The start- and end-time of every cortical wave were defined as first appearance of saturated pixels and the full disappearance of saturated pixels. The absolute maximum spatial extent of every cortical wave was identified and used to align the individual cortical waves of all animals. The area of saturated pixels was then acquired for every CSD wave for every animal at the endpoint of the shortest wave (which ended 37 seconds after the aligned absolute maximum). The area of saturated pixels was groupwise depicted as overlay of the area of every CSD wave upon the general mask.

\section{Statistical analysis}

All quantitative assessment was performed in a blinded manner and based on power calculation wherever it was possible. Based on the type and distribution of data populations (examined with Shapiro-Wilks W test) we applied appropriate statistical tests: in case of two independent groups of data unpaired t-test or Mann Whitney U-test, for two dependent groups of data Wilcoxon signed-rank test, for multiple comparisons one-way ANOVA (with Tukey's test) or Kruskal-Wallis test was used. Statistical analysis was performed with the Statistica 13.4.0.14 package (TIBCO), differences with $p<0.05$ were considered significant throughout this study. 


\section{Supplementary Text for Main Figures}

\section{Figure 1.}

(C) somatic vs dendritic contact lifetimes: $p<0.001, n=26$ contacts from 3 mice.

(F) $n=443$ neurons and 1183 synapses in 4 mice, and 170 neurons in 3 human patients.

(H) $\mathrm{p}<0.001$ in both cases, $\mathrm{n}=114$ and 107 ROIs from 4 mice.

(I) $n=111$ junctions from 4 mice.

(J) $n=50$ cells.

Scale bars: $5 \mu \mathrm{m}$ on $\mathrm{B}, 20 \mu \mathrm{m}$ on $\mathrm{E}, 2 \mu \mathrm{m}$ on $\mathrm{G}, \mathrm{J}$ and $\mathrm{K}$. Median values and interquartile ranges are plotted on $\mathrm{C}$ and $\mathrm{H}$, mean+SD on $\mathrm{F}$ and $\mathrm{I}$, mean+SEM on $\mathrm{K}$.

\section{Figure 2}

(C) Side- and face (90 degrees rotated) view of the 3D model of a contact site. Immunogold density is the highest where the intercellular distance is the smallest.

(D) Density versus distance analysis performed on 3D models of contact sites confirms that the density of $\mathrm{P} 2 \mathrm{Y} 12 \mathrm{R}$-immunogold particles within the junction is significantly higher where the distance between neuronal and microglial membrane is smaller than $13 \mathrm{~nm}(\mathrm{p}<0.001$, MWU test, $\mathrm{n}=13055$ points from 3 neocortical contacts, 3 mice). Median values and interquartile ranges are shown.

(E) Different views of the 3D models of two junctions illustrate that P2Y12R-immunogold density shows an uneven distribution along microglial membranes, being strongly and selectively enriched where the processes are in direct contact with neuronal somata (microglial membrane contacting neuronal cell body - "Somatic", microglial membrane contacting profiles other than neuronal somata - "Nonsomatic $\left.{ }^{\prime \prime}\right)$. $(p<0.001, M W U$ test, $n=24$ surfaces, neocortex, 3 mice).

(G) TOM20 immunofluorescent intensity is significantly higher along neuronal membrane parts where microglial processes contact the somata ( $p<0.001$, MWU test, $n=14,2$ mice).

(I) VNUT immunofluorescent intensity is significantly higher in neurons where microglial processes contact the somata. ( $p=0.002$, MWU test, $n=15,2$ mice).

Scale bars: $500 \mathrm{~nm}$ on $A, 100 \mathrm{~nm}$ on $\mathrm{B}, 5 \mu \mathrm{m}$ on $\mathrm{F}, 3 \mu \mathrm{m}$ on $\mathrm{G}$ and I, $300 \mathrm{~nm}$ on $\mathrm{H}$. Median values and interquartile ranges are plotted on $\mathrm{D}, \mathrm{E}, \mathrm{G}$ and $\mathrm{I}$.

\section{Figure 3}

(B) Contact lifetime was 29 min (median, 10-41 interquartile, $\mathrm{n}=25$ contacts on 19 neurons from 3 mice).

(I) The measured diameter of the released puncta can correspond to sub-diffraction sized emitters because of the point-spread function size ( 0.3-0.4 $\mu \mathrm{m}$ at $488 \mathrm{~nm})$, meaning that the size of the released vesicles can be as small as $40 \mathrm{~nm}$.

(J) $\mathrm{ACSF} / \mathrm{KCl}$ vs ACSF/ACSF $\mathrm{p}=0.0218$, MWU test, $\mathrm{n}=11$ measurements; $\mathrm{SC} / \mathrm{KCl}$ vs $\mathrm{ACSF} / \mathrm{KCl} \mathrm{p}=0.6532$, $\mathrm{MWU}$ test, $\mathrm{n}=11 ; \mathrm{SC}+\mathrm{NIM} / \mathrm{KCl}$ vs $\mathrm{ACSF} / \mathrm{KCl} p=0.0271, \mathrm{n}=10 ; \mathrm{SC}+\mathrm{CLO} / \mathrm{KCl}$ vs $\mathrm{ACSF} / \mathrm{KCl} p=0.0284, \mathrm{n}=10$. 
Scale bars: $2 \mu \mathrm{m}$ on $A$ and $K, 4 \mu \mathrm{m}$ on $\mathrm{B}, 10 \mu \mathrm{m}$ on $\mathrm{C}$ and $\mathrm{D}, 500 \mathrm{~nm}$ on $\mathrm{F}, 3 \mu \mathrm{m}$ on $\mathrm{G}$, and $200 \mathrm{~nm}$ on the $\mathrm{EM}$ insert on $\mathrm{K}$. Median values and interquartile ranges are plotted on I, mean+SD on E, mean+SEM on J. 


\section{Figure 4}

(A) Baseline in vivo $2 \mathrm{P}$ imaging of cortical microglia-neuron contacts of tdTomato electroporated CX3CR1 ${ }^{+/ G F P}$ mice was followed by administration of the P2Y12R-inhibitor PSB0739 (PSB) into the cisterna magna (i.c.m.) and a further imaging session.

(C) ctrl som. vs. PSB som. $\mathrm{p}=0.0331$, MWU test, $\mathrm{n}=12$ contacts for $\mathrm{ctrl}$ som., 14 ctrl dendr., 15 PSB som., 13 PSB dendr. from 2 mice.

(D) ctrl vs CNO+/cFos+ $p=0.0139$; ctrl vs $\mathrm{CNO}+$ /cFos- cells, $\mathrm{p}=1$, Kruskal-Wallis test, $\mathrm{n}=101$ cells from 8 mice.

(E) ctrl vs CNO+/cFos+ $\mathrm{p}=0.7497$; ctrl vs $\mathrm{CNO}+$ /cFos- cells, $\mathrm{p}=1$, Kruskal-Wallis test, $\mathrm{n}=85$ cells from 6 mice.

(G) ( $p<0.001, \mathrm{MWU}$ test, $\mathrm{n}=124$ cells from 6 mice).

Scale bars: $5 \mu \mathrm{m}$ on $B$ and $G, 8 \mu \mathrm{m}$ on $\mathrm{D}$ and $\mathrm{E}$. Median values and interquartile ranges are plotted on $\mathrm{C}$, $D, E$ and $G$.

\section{Figure 5}

(A) $p<0.001, n=189$ mitochondria.

(B) $p<0.001, n=30$ neurons.

(D) ctrl vs. stroke: $p<0.001$, PSB ctrl vs. PSB stroke: $p=0.792$, diazo. ctrl vs. diazo. stroke: $p=0.053$, MWU test, $n=140$ neurons

(E) $p=0.007$, MWU test, $n=113$ and 136 neurons from 3 control and 3 stroke patients, respectively

(F) $p=0.0439$, unpaired t-test, $n=9$ control and 8 PSB-treated mice

(G) left panel: Individual ROI-to-GC scores were normalized to baseline and quantified for group comparison ( $p=0.0077$, unpaired t-test, $n=4$ control and 3 PSB-treated mice).

(H) $\mathrm{p}<0.0001, \mathrm{n}=96$ neurons from 3 mice

(I) Infarct volumes: stroke: $14.96 \pm 0.95 \mathrm{~mm}^{3}$; stroke+PSB: $23.06 \pm 2.519 \mathrm{~mm}^{3}, \mathrm{p}=0.008$, MWU test, $\mathrm{n}=10$ control and 10 stroke mice

Scale bars: $4 \mu \mathrm{m}$ on $\mathrm{A}, 2 \mu \mathrm{m}$ on $\mathrm{B}, 8 \mu \mathrm{m}$ on $\mathrm{E}, 20 \mu \mathrm{m}$ on $\mathrm{H}$. Median values and interquartile ranges are plotted on $A, B, D$ and $E$, mean+SEM on F, G, H and I. 

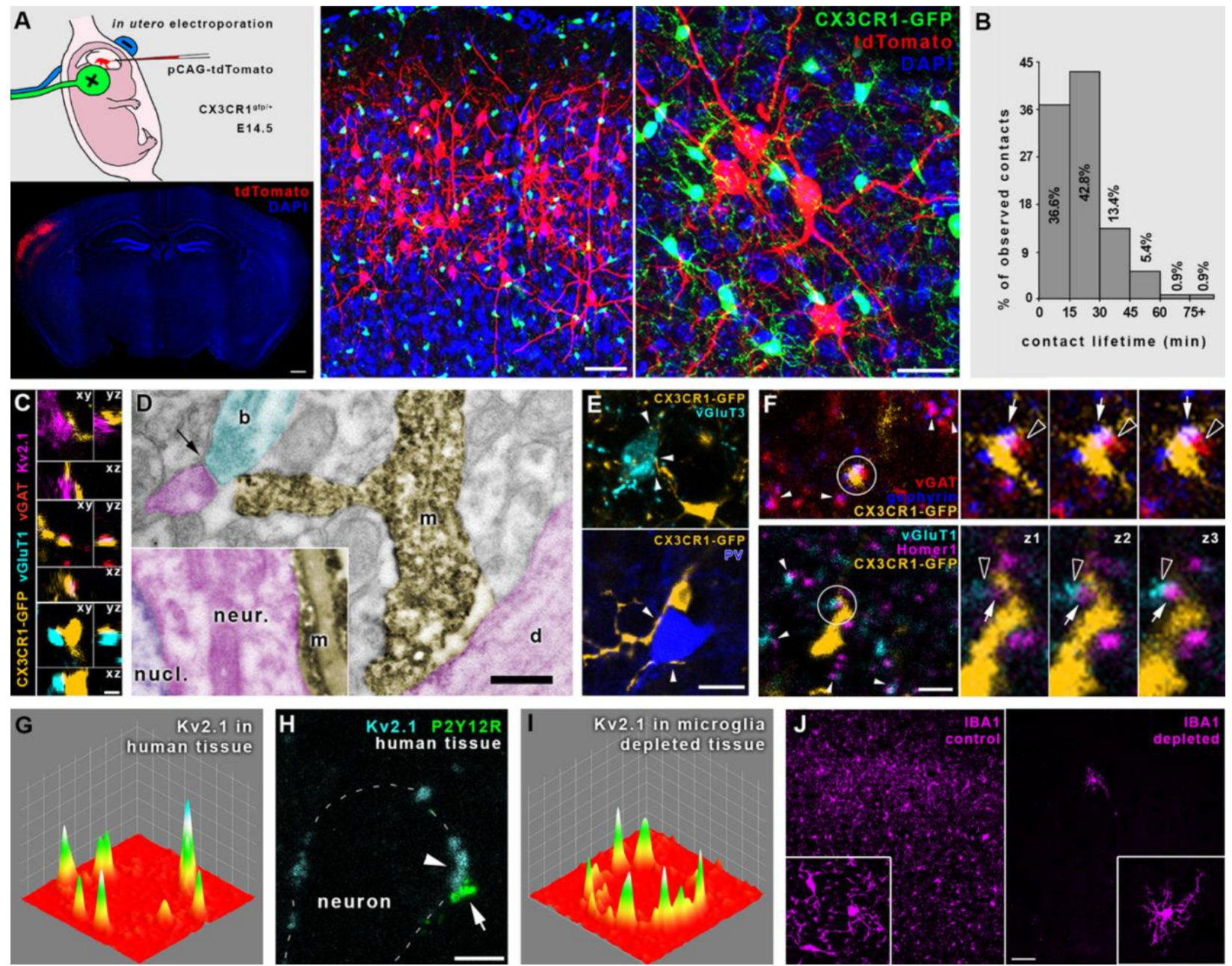

Fig. S1.

(A) Result of CX3CR1 ${ }^{+/ G F P}$ mouse in utero electroporation with pCAG-IRES-tdTomato. Lower left panel shows the spread of tdTomato-positive neurons in red. Middle and right panels show the nonoverlapping staining of electroporated neurons (red) and microglia (green). Cell nuclei are visible in blue. (B) Distribution of microglial contact lifetimes observed with in vivo $2 \mathrm{P}$ imaging $(\mathrm{n}=112$ contacts from 2 mice). (C) Orthogonal projections show microglial (yellow) contacts with neuronal cell body (magenta), GABAergic (red) and glutamatergic (cyan) synapses. Images represent contacts marked on Fig. 1D. (D) Electron microscopic images showing microglial processes $(\mathrm{m})$ establishing direct contact with neuronal cell body (neur.), dendritic shaft (d) and presynaptic bouton (b) in mice. Microglia are visualized by immunoperoxidase reaction against Iba1. Pseudocoloring shows microglia in yellow, neuronal cell body and dendritic processes in magenta, presynaptic bouton in cyan and nucleus (nucl.) in purple. Arrow points at a dendritic spine receiving asymmetric synaptic contact. (E) CLSM image planes show microglial processes (yellow) touching cell bodies of hippocampal vGluT3+ (cyan) and PV+ (blue) interneurons. (F) Microglial processes contacting cortical inhibitory and excitatory synapses. Presynaptic terminals are visible by stainings against vesicular GABA transporter (vGAT, red) and type 1 vesicular glutamate transporter (vGlut1, cyan), while postsynaptic side is characterized by stainings against gephyrin (blue) and Homer1 (magenta). White arrowheads point at colocalization of pre- and postsynaptic markers. Microglial contacts in white circles are enlarged in the panels right, with the previous and following Zplanes from the stack $(Z$-step $=250 \mathrm{~nm})$. Empty arrowheads point at the presynaptic marker, white 
arrows point at the postsynaptic side. (G) Heatmap shows Kv2.1 clusters of a human cortical neuron. (H) CLSM image shows that a P2Y12R-labeled microglial process contacts a human cortical neuron exactly at the spot where a Kv2.1 cluster is located. (I) Heatmap shows that Kv2.1-clustering of mouse cortical neurons is not affected by microglia depletion. (J) CLSM images show that 3 week PLX5622-diet almost completely depleted microglia from the brain. Scale bars: $500 \mu \mathrm{m}$ on left panel of $A, 50 \mu \mathrm{m}$ on middle panel of $A$ and on J, $25 \mu \mathrm{m}$ on right panel of $A, 1 \mu \mathrm{m}$ on $C$ and $D, 15 \mu \mathrm{m}$ on $E, 2 \mu \mathrm{m}$ on $\mathrm{F}$ and $\mathrm{H}$. 

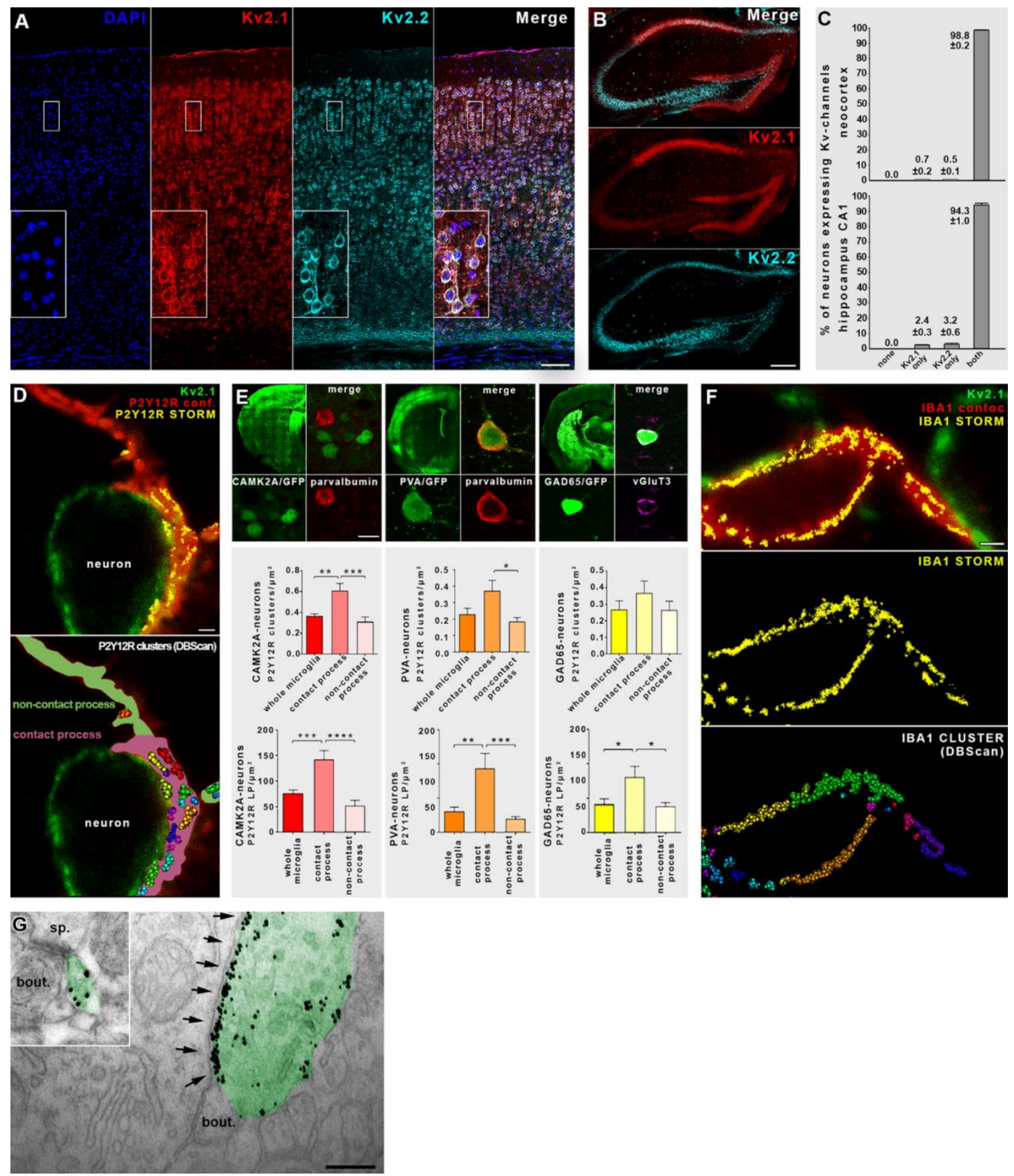

Fig. S2.

(A) CLSM images show labeling patterns of Kv2.1 and Kv2.2 in the neocortex. (B) CLSM images show labeling patterns of Kv2.1 and Kv2.2 in the hippocampus. (C) Percentages of neurons expressing Kvchannels in the neocortex and hippocampus. (D) P2Y12R clustering differs depending on location. Microglial processes were classified depending on neuronal contacts established: contact processes are pale magenta, non-contact processes are pale green on bottom panel. (E) Analysis of P2Y12R cluster and LP density on different microglial processes contacting distinct cell populations. Microglial contact 
processes on CamK2a (red) and PVA-positive cells (orange) possess significantly higher cluster density, than non-contact segments, while there is a similar trend observable in the case of GAD65-positive cells (yellow, for detailed results see Supplementary Table 1.). Contact processes had significantly higher number of P2Y12R LPs in all populations. Anti-PV and vGluT3 labelings were used to confirm cell specificity. (F) Super-resolution imaging of microglial lba1 shows no clustering at somatic junctions. CLSM images show neuronal Kv2.1 (green) and a contacting microglial process made visible by Iba1labeling (red). Iba1 (yellow) STORM-signal is overlaid and shown individually in the middle panel. Lower panel shows a homogenous distribution of Iba1 LPs using DBScan analysis. (G) Contact dependent clustering of $\mathrm{P} 2 \mathrm{Y} 12 \mathrm{Rs}$ is absent at synapses as confirmed by immunogold-electron microscopy. Scale bars: $100 \mu \mathrm{m}$ on $A$ and $B, 1 \mu \mathrm{m}$ on $D$ and $F, 10 \mu \mathrm{m}$ on $E, 250 \mathrm{~nm}$ on $\mathrm{G}$. Meantrange is plotted on $C$, Mean+SEM is plotted on $\mathrm{E}$. 

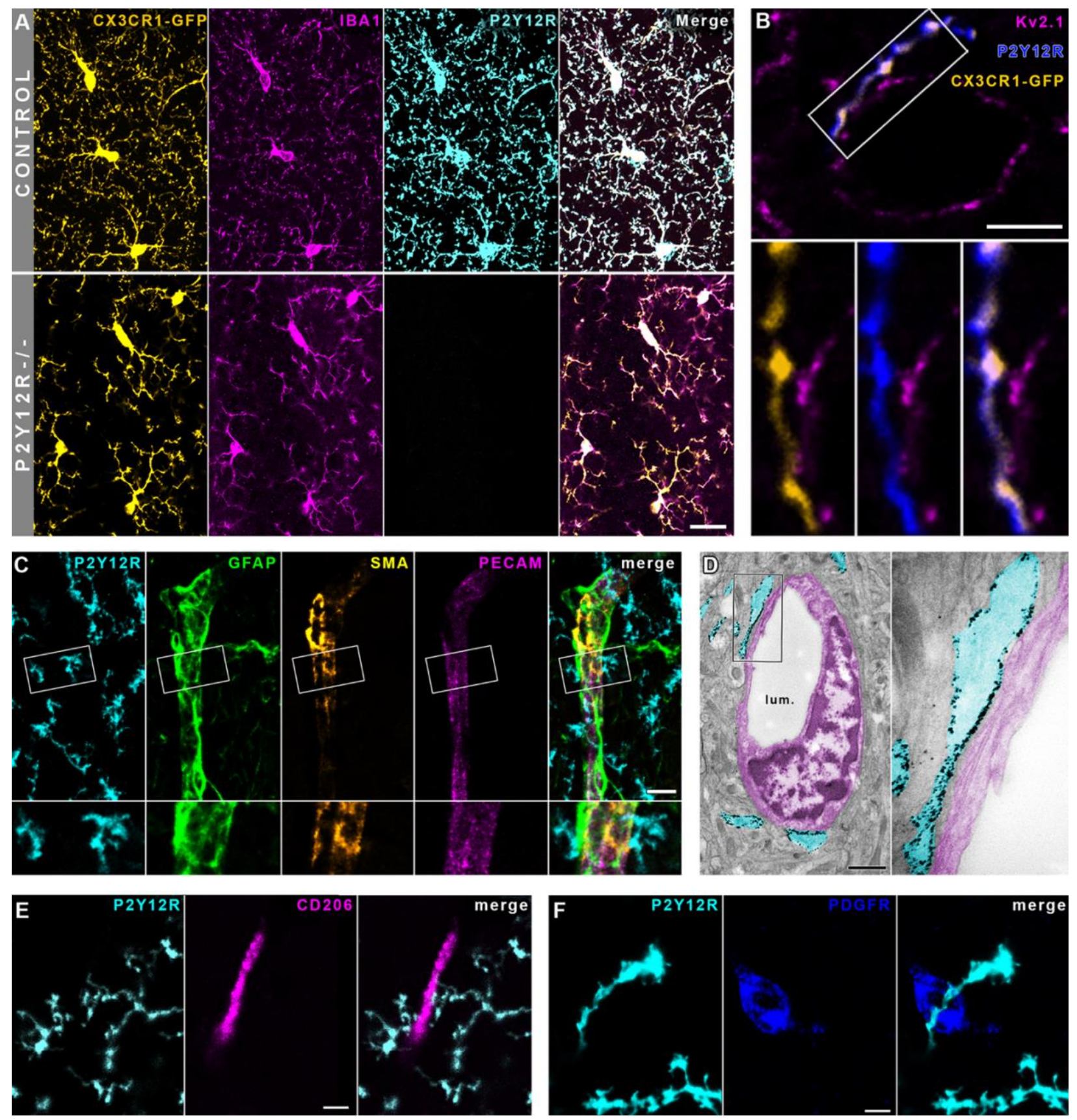

Fig. S3.

(A) CLSM images show triple immunolabeling for GFP, Iba1 and P2Y12R in CX3CR1 ${ }^{+/ G F P}$ and CX3CR1 ${ }^{+/ G F P}-$ P2Y12R ${ }^{-/}$animals. (B) CX3CR1-GFP and P2Y12R double positive microglial processes touch Kv2.1 clusters on neuronal somatic membrane. (C) P2Y12R-positive microglial processes contact cells of the neurovascular unit. (D) Transmission electron micrographs show P2Y12R-immunogold labeled microglial processes (cyan pseudocolour) touching vascular endothelial cell (magenta pseudocolour). P2Y12R expression is clearly restricted to microglia. (E) P2Y12R-labeled microglial processes are contacting CD206-positive perivascular macrophages (PVM). PVMs do not express P2Y12Rs. (F) P2Y12R-labeled microglial processes are contacting PDGFRß-positive pericytes. Pericytes are not expressing P2Y12Rs. Scale bars: $20 \mu \mathrm{m}$ on $\mathrm{A}, 4 \mu \mathrm{m}$ on $\mathrm{B}, 10 \mu \mathrm{m}$ on $\mathrm{C}, 1 \mu \mathrm{m}$ on $\mathrm{D}, 5 \mu \mathrm{m}$ on $\mathrm{E}, 3 \mu \mathrm{m}$ on $\mathrm{F}$. 

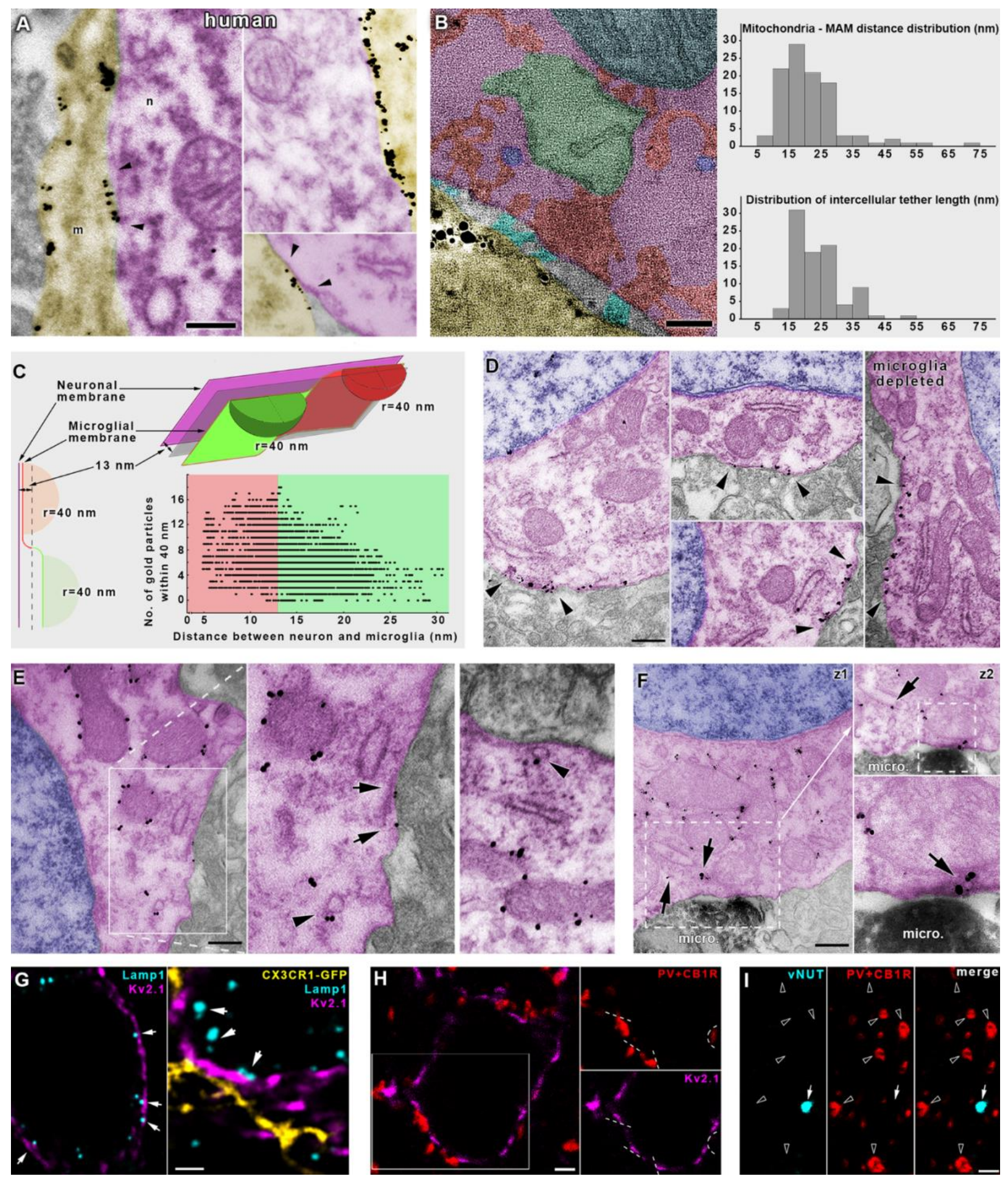

\section{Fig. S4.}

(A) Electron microscopic image shows a microglial process $(\mathrm{m})$ establishing direct contact with neuronal cell body $(n)$ in human tissue. Microglia is identified by immunogold-labeling against P2Y12R. Arrowheads point at accumulation of gold particles at contact sites. (B) Single virtual plane of an electron tomographic volume (thickness: $0.49 \mathrm{~nm}$ ) showing a P2Y12R-positive (black immunogold particles) microglial process (yellow) contacting a neuronal cell body (magenta). Mitochondria (blue), mitochondria-associated membranes (MAM, green), vesicles (purple) and intracellular densities (red) 
are commonly visible in the vicinity of these junctions. In some cases, intercellular linking structures (cyan) can be seen within the cleft. Right: distribution of measured distances between mitochondria and MAM (upper chart, $n=105$ ) and measured length of intercellular tethers (lower chart, $n=89$ ). (C) Schematic illustration showing the principles of P2Y12R density and membrane distance measurements using electron tomography (Fig. 2C, D). Single points of the microglial membrane were divided into two groups based on their closest distance from the neuronal membrane: closer than the average distance of $13 \mathrm{~nm}$ (red) or farther (green). For every observed point, P2Y12R labeling gold particles were counted within a radius of $40 \mathrm{~nm}$. In the bottom right corner, the distribution of measured points is shown in the case of a representative junction. (D) Transmission electron micrographs show Kv2.1 clusters on cortical neuronal cell bodies (arrowheads). Kv2.1-clustering and the association of the cluster with neuronal organelles is not affected by microglia depletion (right panel). (E) Transmission electron micrographs show TOM20-immunogold labeling in neocortical neurons. Immunogold labeling (black grains) is specifically associated with outer mitochondrial membranes, while TOM20-positive vesicles can also be observed (arrowheads). Some immunogold particles can be found on the plasma membrane of the neurons (arrows), suggesting the exocytosis of mitochondria-derived vesicles. Nucleus is blue, neuronal cytoplasm is magenta. Left and middle panel is the same as on Fig. $2 \mathrm{H}$. (F) Electron micrographs from TOM20-lba1 combined immunogold-immunoperoxidase reaction. Right upper panel is taken from a consecutive serial section and corresponds to the dashed area on the left panel. Right lower panel is enlarged from the right upper. Iba1-positive (dark DAB precipitate) microglial process contacts the cell body of a cortical pyramidal cell. TOM20-immunogold signal decorates the outer mitochondrial membrane, while arrows point to smaller membrane structures (putative MDVs), also positive for TOM20. Note the presence of a TOM20 positive vesicle attached to the neuronal plasmamembrane within the somatic junction on the right lower panel. (G) CLSM images show Lamp1-positive neuronal lysosomes closely apposed to neuronal Kv2.1-clusters. Right panel shows a microglial process touching a neuronal Kv2.1-cluster with closely located/attached Lamp1-positive lysosomes. (H) Kv2.1 accumulation does not overlap with somatic inhibitory terminals. CLSM images show perisomatic terminals stained with antibodies against cannabinoid receptor type 1 (CB1R) and PV (red) and neuronal Kv2.1 (magenta). Panels on the right show the area in white rectangle on the first image. Dashed lines mark clear separation between perisomatic boutons and Kv2.1-signal, $n=220$ perisomatic boutons tested for Kv2.1 from two mice. (I) Neuronal vNUT signal (cyan) is not present in perisomatic inhibitory terminals (red), $\mathrm{n}=194$ perisomatic boutons tested for vNUT from two mice. Scale bars: $300 \mathrm{~nm}$ on A (400 for right bottom panel), $50 \mathrm{~nm}$ on $\mathrm{B}, 300 \mathrm{~nm}$ on $\mathrm{D}, \mathrm{E}$ and $\mathrm{F}, 1 \mu \mathrm{m}$ on $\mathrm{G}(2 \mu \mathrm{m}$ for left panel), $2 \mu \mathrm{m}$ on $\mathrm{H}$ and I. 

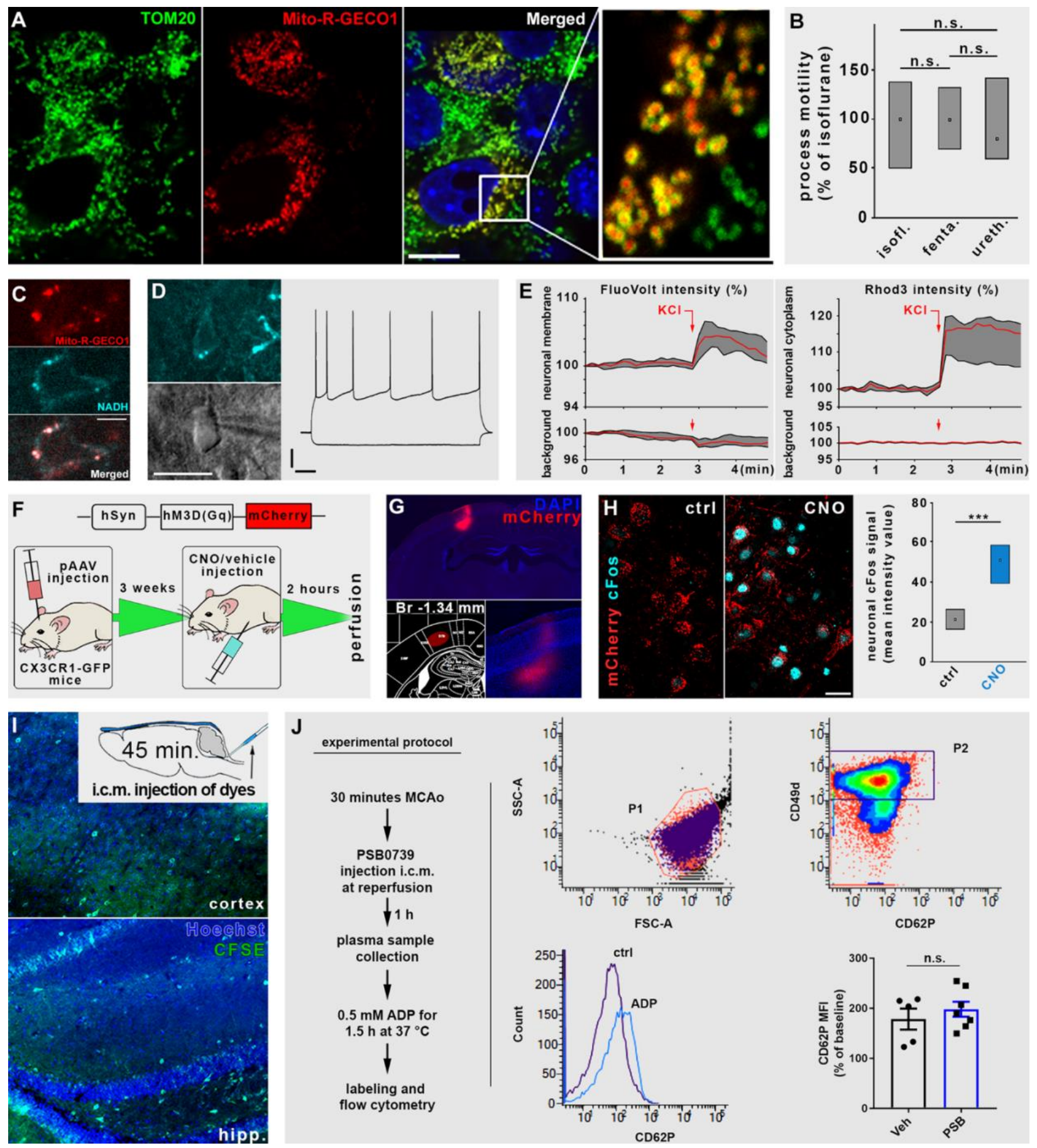

\section{Fig. S5.}

(A) Neuronal cell culture transfected with CMV-Mito-R-Geco1 and counterstained with TOM20 and DAPI. (B) Effects of different anaesthetics on microglial process motility. Median process motility observed: isoflurane $0.6 \mu \mathrm{m} / \mathrm{min}, 0.3-0.83$ interquartile; fentanyl $0.6 \mu \mathrm{m} / \mathrm{min}, 0.42-0.78$; urethane 0.48 $\mu \mathrm{m} / \mathrm{min}, 0.36-0.84 ; \mathrm{n}=153$ processes from 9 animals. (C) Mito-R-Geco1 expression colocalizes with $\mathrm{NADH}$ intrinsic fluorescence. (D) Representative NADH intrinsic fluorescence image of a pyramidal cell. Left: Sample image of NADH fluorescence of neuronal somata. Middle: DIC image of the same region with whole cell patch clamp electrode. Right: Electrophysiological recordings of membrane potential response to -100 and +160 pA current injections. Scales: $20 \mathrm{mV}$ and $100 \mathrm{~ms}$. (E) $40 \mathrm{mM} \mathrm{KCl}$ induces rapid 
membrane depolarization and calcium influx in cultured neurons in vitro. (F) Outline of chemogenetic experiments. (G) An example of an injection site. (H) CNO administration induced a 2.3-fold increase in cFos mean fluorescent intensity value in DREADD expressing neurons ( $<<0.001, n=50-50$ cells from 3 vehicle and 3 CNO-treated mice). (I) Mixture of carboxyfluorescein succinimidyl ester (CFSE) and Hoechst injected into the cisterna magna (i.c.m.) rapidly diffuses to all layers of neocortex and hippocampus. (J) Central blockade of microglial P2Y12R with PSB does not alter ADP-induced platelet activation. Platelet rich plasma samples were collected from mice $1 \mathrm{~h}$ after $30 \mathrm{~min}$ MCAo and stimulated ex vivo with $0.5 \mathrm{mM}$ ADP for $1.5 \mathrm{~h}$ at $37^{\circ} \mathrm{C}$. Platelets shown on SSC x FSC dot blots were labeled with antiCD49d PE and anti-CD62P APC. ADP-induced increases in platelet CD62P were not altered in mice treated with i.c.m. PSB compared to vehicle-treated animals ( $n=5$ vehicle-treated and 7 PSB-treated samples). Unpaired t-test, n.s. - not significant. Scale bars: $10 \mu \mathrm{m}$ on A, $5 \mu \mathrm{m}$ on C, $25 \mu \mathrm{m}$ on $\mathrm{D}, 20 \mu \mathrm{m}$ on $\mathrm{G}$. Median and interquartile ranges are plotted on $\mathrm{B}, \mathrm{E}$ and $\mathrm{H}$, mean+SEM is plotted on $\mathrm{J}$. 

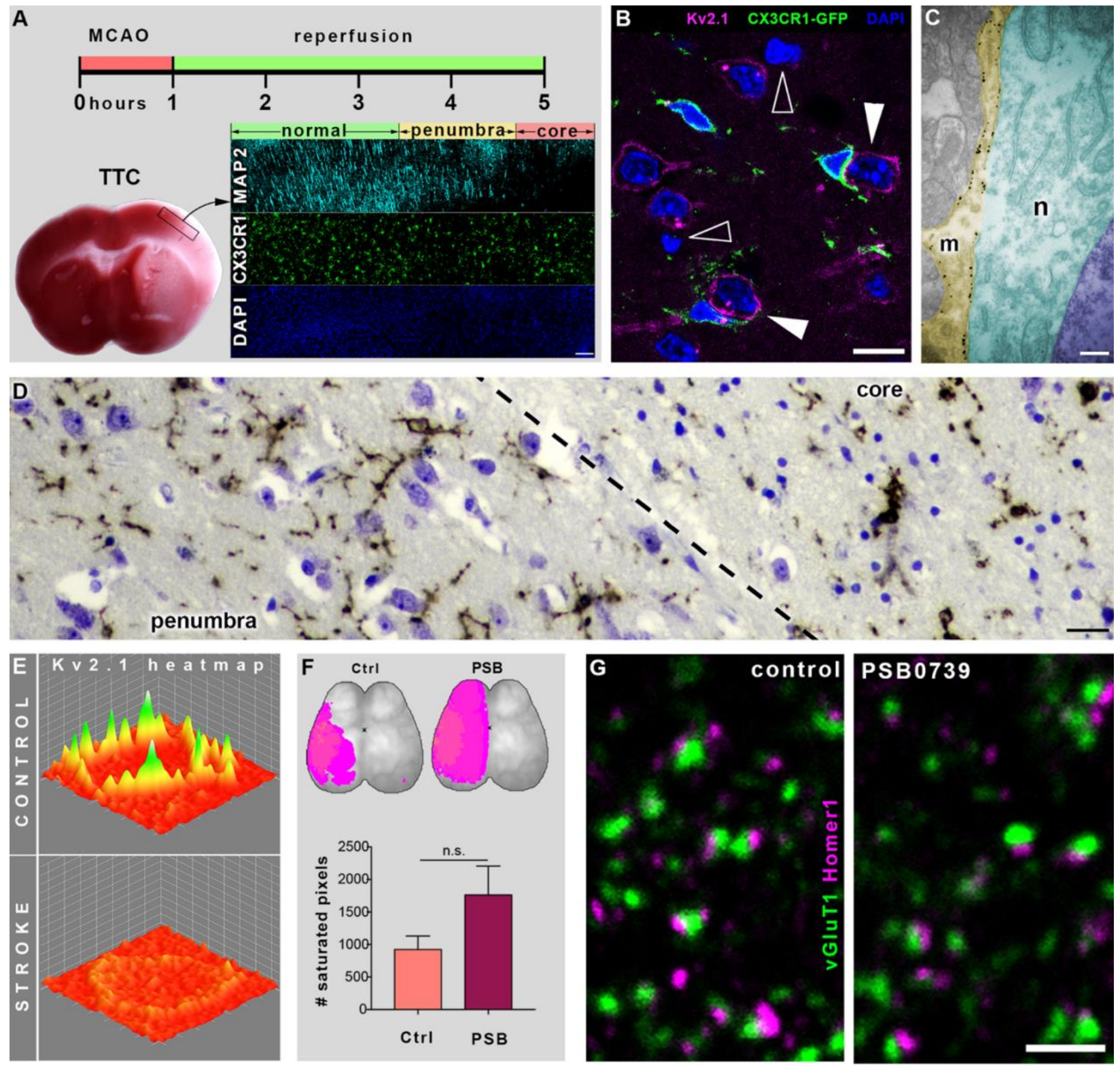

Hehic. or PSB0739 injec-
veris tion into cisterna magna injection capillary

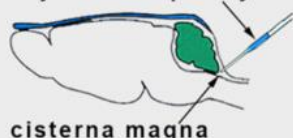

cisterna magna
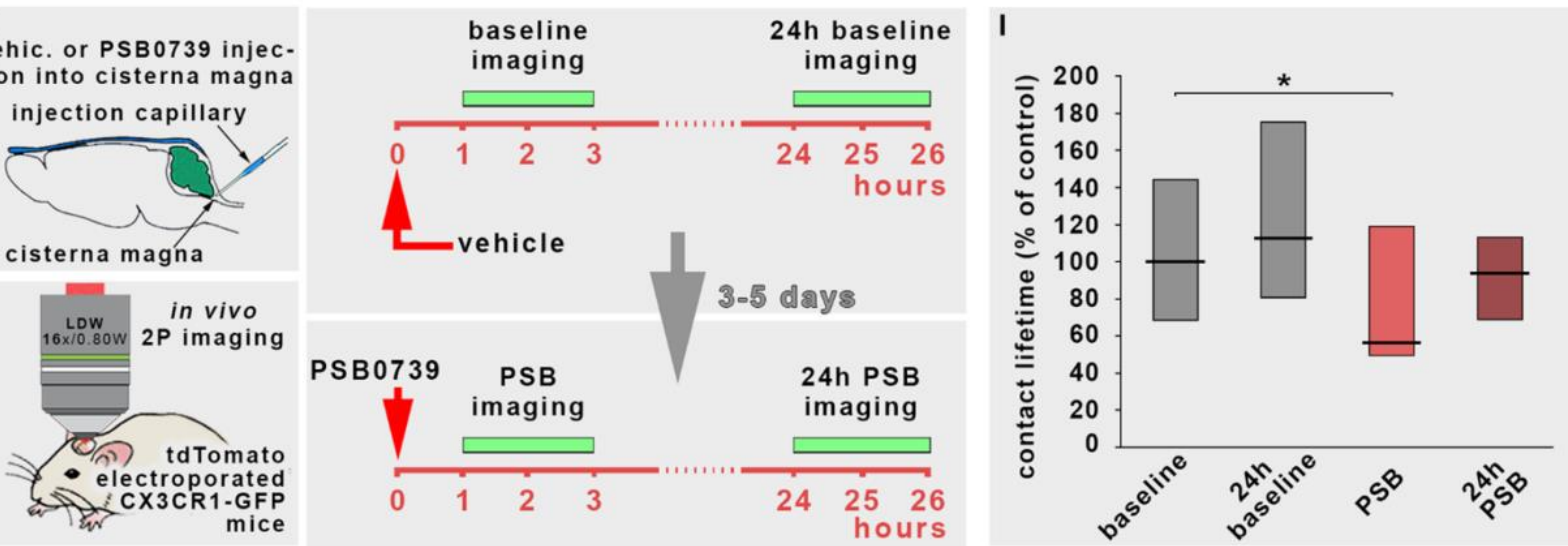

Fig. S6.

(A) Outline of middle cerebral artery occlusion (MCAo) experiment and delineation of core/penumbra regions of stroke affected brain. 1 hour MCAo was followed by 4 hours of reperfusion. Delineation was 
performed with the use of TTC staining and immunofluorescent labeling of MAP2 and microglia. (B) DAPI staining pattern reveals that neurons with increased microglial coverage (white arrowheads) in the penumbra region possess normal chromatin structure, confirming their viability. Empty arrowheads point to pycnotic nuclei. (C) Transmission electron micrograph shows a P2Y12R-immunogold particle labeled microglial process (yellow pseudocolour) covering a neuron in the penumbra region. Neuronal membrane integrity and chromatin structure are sustained. (D) Delineation of core/penumbra border in postmortem human cortical tissue. Neurons and nuclei are visualized by Nissl-staining (blue), microglia is labeled by anti-P2Y12R DAB-Ni immunoperoxidase reaction (dark brown precipitate). (E) Heatmaps of Kv2.1 immunostaining reveal that Kv2.1 clusters disappear after stroke in the penumbra. (F) The saturated calcium signal after experimental stroke (left MCAo in every experiment) corresponds to the dysfunctional tissue during the occlusion. The maps show an overlay of the area of saturated pixels (pink) of every animal in the two groups ( $n=9$ control and 8 PSB-treated mice). (G) CLSM images show vGlut1 and Homer1 immunolabeling after acute i.c.m. administration of vehicle (control) or PSB. The closely apposed vGluT1 and Homer1 puncta represent glutamatergic synaptic contacts. PSB-treatment did not alter neocortical synapse numbers $\left(0.353\right.$ synapse $/ \mu \mathrm{m}^{2}$ in control, and 0.352 synapse $/ \mu \mathrm{m}^{2}$ in PSB-injected, $n=423$ appositions from 4 animals). (H) Schematic outline of repeated 2P imaging of PSB effects. Vehicle was administered i.c.m., and baseline imaging performed between the 1st and 3rd hours after injection. The imaging session was repeated after $24 \mathrm{~h}$ (24h baseline). After 3-5 days, PSB was injected i.c.m., and imaging performed between the 1st and 3rd hours after injection (PSB). The imaging session was repeated after 24h (24h PSB). (I) Acute central PSB administration decreased the contact lifetime to $56 \%$ of control, but this effect could not be observed after 24 hours (68.8-143.8\% baseline interquartile range, $112.5 \%$ (81.3-175) $24 \mathrm{~h}$ baseline, 56.3\% (50-118.8) after PSB, 93.8 (68.8-112.5) 24h after PSB; $p=0.0139, n=173$ contacts from 3 mice). Scale bars: $100 \mu \mathrm{m}$ on $A, 10 \mu \mathrm{m}$ on $B, 300 \mathrm{~nm}$ on C, 12 $\mu \mathrm{m}$ on $\mathrm{D}, 2 \mu \mathrm{m}$ on $\mathrm{G}$. Mean+SEM is plotted on $\mathrm{F}$, median and interquartile ranges on I. 
Table S1.

Statistical data for analyses depicted on Figures $1 \mathrm{~K}$ and S2E.

\begin{tabular}{|c|c|c|c|c|c|c|}
\hline \multicolumn{7}{|c|}{ Localisation point density (LP/um2) } \\
\hline animal & category & mean & S.E.M. & $\mathrm{n}$ & comparison (Tukey's multiple comparisons test) & $\mathrm{p}$-value \\
\hline \multirow{3}{*}{ GCamp6-injected } & contact & 136.8 & 10.02 & 12 & contact vs. non-contact & $<0,0001$ \\
\hline & non-contact & 60.42 & 7.719 & 12 & contact vs. whole microglia & $<0,0001$ \\
\hline & whole microglia & 76.8 & 5.128 & 12 & whole microglia vs. non-contact & 0.3179 \\
\hline \multirow{3}{*}{ camK2A/gfp/22 } & contact & 142.6 & 17.17 & 18 & contact vs. non-contact & $<0,0001$ \\
\hline & non-contact & 52.2 & 10.08 & 17 & contact vs. whole microglia & 0.0008 \\
\hline & whole microglia & 76.47 & 6.054 & 18 & whole microglia vs. non-contact & 0.3423 \\
\hline \multirow{3}{*}{ gad65_3e/gfp 5.5/30 } & \begin{tabular}{|c|} 
contact \\
\end{tabular} & 81.05 & 15.37 & 11 & contact vs. non-contact & 0.0116 \\
\hline & \begin{tabular}{|c|} 
non-contact \\
\end{tabular} & 38.62 & 4.892 & 13 & contact vs. whole microglia & 0.0197 \\
\hline & whole microglia & 41.58 & 7.661 & 13 & whole microglia vs. non-contact & 0.973 \\
\hline \multirow{3}{*}{ BAC_pva/gfp/2 } & \begin{tabular}{|c|} 
contact \\
\end{tabular} & 93.75 & 21.59 & 10 & contact vs. non-contact & 0.0009 \\
\hline & non-contact & 20.03 & 2.746 & 10 & contact vs. whole microglia & 0.003 \\
\hline & whole microglia & 31.17 & 5.736 & 12 & whole microglia vs. non-contact & 0.7957 \\
\hline \multicolumn{7}{|c|}{ Cluster density (cluster/um2) } \\
\hline animal & category & mean & S.E.M. & $\mathrm{n}$ & comparison (Tukey's multiple comparisons test) & p-value \\
\hline \multirow{3}{*}{ GCamp6-injected } & contact & 0.9705 & 0.084 & 12 & contact vs. non-contact & $<0,0001$ \\
\hline & non-contact & 0.4451 & 0.071 & 12 & contact vs. whole microglia & 0.0004 \\
\hline & whole microglia & 0.5401 & 0.055 & 12 & whole microglia vs. non-contact & 0.6164 \\
\hline \multirow{3}{*}{ camK2A/gfp/22 } & contact & 0.6089 & 0.069 & 18 & contact vs. non-contact & 0.0002 \\
\hline & \begin{tabular}{|c|} 
non-contact \\
\end{tabular} & 0.3111 & 0.046 & 18 & contact vs. whole microglia & 0.0031 \\
\hline & whole microglia & 0.3673 & 0.02 & 18 & whole microglia vs. non-contact & 0.7007 \\
\hline \multirow{3}{*}{ gad65_3e/gfp 5.5/30 } & contact & 0.368 & 0.072 & 9 & contact vs. non-contact & 0.4608 \\
\hline & non-contact & 0.2659 & 0.052 & 13 & contact vs. whole microglia & 0.4681 \\
\hline & whole microglia & 0.2684 & 0.051 & 14 & whole microglia vs. non-contact & 0.9994 \\
\hline \multirow{3}{*}{ BAC_pva/gfp/2 } & contact & 0.3726 & 0.062 & 10 & contact vs. non-contact & 0.0107 \\
\hline & non-contact & 0.1839 & 0.024 & 11 & contact vs. whole microglia & 0.0553 \\
\hline & whole microglia & 0.2293 & 0.036 & 12 & whole microglia vs. non-contact & 0.7145 \\
\hline
\end{tabular}


Table S2.

Data of human patients.

\begin{tabular}{|c|c|c|c|c|c|c|c|c|}
\hline Subject & Code & Gender & $\begin{array}{c}\text { Age } \\
\text { (years) }\end{array}$ & $\begin{array}{l}\text { Health } \\
\text { status }\end{array}$ & $\begin{array}{c}\text { Survival } \\
\text { after } \\
\text { stroke } \\
\text { (days) }\end{array}$ & Comorbidities & $\begin{array}{c}\text { Cause of } \\
\text { death }\end{array}$ & $\begin{array}{c}\text { Tissue } \\
\text { sample } \\
\text { type }\end{array}$ \\
\hline $\begin{array}{l}\text { Stroke } \\
\text { patient }\end{array}$ & 2011/0092 & female & 77 & normal & 2 & $\begin{array}{l}\text { arterial } \\
\text { hypertension, } \\
\text { type II diabetes, } \\
\text { hyperthyreosis }\end{array}$ & stroke & $\begin{array}{l}\text { paraffin } \\
\text { embedded } \\
\text { sections }\end{array}$ \\
\hline $\begin{array}{l}\text { Stroke } \\
\text { patient }\end{array}$ & $2003 / 0029$ & male & 66 & $\begin{array}{c}\text { preceding } \\
\text { systemic } \\
\text { inflammatory } \\
\text { burden }\end{array}$ & 1 & $\begin{array}{c}\text { arterial } \\
\text { hypertension }\end{array}$ & stroke & $\begin{array}{l}\text { paraffin } \\
\text { embedded } \\
\text { sections }\end{array}$ \\
\hline $\begin{array}{l}\text { Stroke } \\
\text { patient }\end{array}$ & $2014 / 0050$ & female & 78 & normal & 1 & unknown & stroke & $\begin{array}{l}\text { paraffin } \\
\text { embedded } \\
\text { sections }\end{array}$ \\
\hline $\begin{array}{l}\text { Control } \\
\text { subject }\end{array}$ & SKO3 & female & 59 & normal & n.a. & $\begin{array}{c}\text { ischemic } \\
\text { cardiomyopathy }\end{array}$ & $\begin{array}{l}\text { cardiogenic } \\
\text { shock }\end{array}$ & $\begin{array}{c}\text { free } \\
\text { floating and } \\
\text { paraffin } \\
\text { sections }\end{array}$ \\
\hline $\begin{array}{l}\text { Control } \\
\text { subject }\end{array}$ & SKO13 & female & 60 & normal & n.a. & $\begin{array}{l}\text { chronic } \\
\text { bronchitis }\end{array}$ & $\begin{array}{l}\text { respiratory } \\
\text { arrest }\end{array}$ & $\begin{array}{c}\text { free } \\
\text { floating and } \\
\text { paraffin } \\
\text { sections }\end{array}$ \\
\hline $\begin{array}{l}\text { Control } \\
\text { subject }\end{array}$ & SKO16 & male & 73 & normal & n.a. & $\begin{array}{c}\text { atherosclerosis, } \\
\text { pneumonia }\end{array}$ & $\begin{array}{l}\text { respiratory } \\
\text { arrest }\end{array}$ & $\begin{array}{c}\text { free } \\
\text { floating and } \\
\text { paraffin } \\
\text { sections }\end{array}$ \\
\hline
\end{tabular}


Table S3.

List of antibodies used in the study.

\begin{tabular}{|c|c|c|c|c|}
\hline Primary antibodies & host & source & catalog $\mathrm{nr}$. & RRID \\
\hline CB1 & goat & MyBioSource & MBS422809 & n.a. \\
\hline CytC & mouse & BioLegend & 612302 & RRID:AB_315774 \\
\hline c-fos & guinea-pig & Synaptic Systems & 226004 & RRID:AB_2619946 \\
\hline CD206 & rat & BioRad & MCA2235 & RRID:AB_324622 \\
\hline CD42d & armenian & eBioScience & $12-0421-80$ & RRID:AB_10804037 \\
\hline CD62-P-APC & mouse & eBioScience & $17-0626-80$ & RRID:AB_11218294 \\
\hline Gephyrin & mouse & Synaptic Systems & 147021 & RRID:AB_2232546 \\
\hline GFAP & chicken & Synaptic Systems & 173006 & RRID:AB_2619873 \\
\hline GFP & chicken & Invitrogen & A10262 & RRID:AB_2534023 \\
\hline Homer1 & chicken & Synaptic Systems & 160006 & RRID:AB_2631222 \\
\hline Homer1 & rabbit & Synaptic Systems & 160003 & RRID:AB_887730 \\
\hline Iba1 & goat & Novusbio & NB100-1028 & RRID:AB_521594 \\
\hline Iba1 & rabbit & Wako Chemicals & $019-19741$ & RRID:AB_839504 \\
\hline Kv2.1 & mouse & NeuroMab & $75-014$ & RRID:AB_10673392 \\
\hline Kv2.1 & rabbit & Synaptic Systems & 231002 & RRID:AB_2131650 \\
\hline Kv2.2 & rabbit & Synaptic Systems & 231103 & RRID:AB_10805652 \\
\hline Lamp1 & rabbit & Abcam & $a b 24170$ & RRID:AB_775978 \\
\hline MAP2 & guniea-pig & Synaptic Systems & 188004 & RRID:AB_2138181 \\
\hline NTPDase1 & rabbit & Labome & rN1-6L & n.a. \\
\hline $\mathrm{P} 2 \mathrm{Y} 12 \mathrm{R}$ & rabbit & Anaspec & AS-55042A & RRID:AB_2267540 \\
\hline P2Y12R & rabbit & Anaspec & AS-55043A & RRID:AB_2298886 \\
\hline Parvalbumin & goat & Swant & PVG 213 & RRID:AB_2721207 \\
\hline PDGFRB & goat & R and D Systems & AF1042 & RRID:AB_2162633 \\
\hline Pecam1 & rat & BioLegend & 102501 & RRID:AB_312908 \\
\hline RFP & rat & ChromoTek & $5 \mathrm{f} 8-100$ & RRID:AB_2336064 \\
\hline SMA & mouse & Abcam & ab7817 & RRID:AB_262054 \\
\hline SMI32 & mouse & Covance & SMI-32P & RRID:AB_10719742 \\
\hline TOM20 & rabbit & Santa Cruz & sc-11415 & RRID:AB_2207533 \\
\hline vGAT & guinea pig & Synaptic Systems & 131004 & RRID:AB_887873 \\
\hline vGluT1 & guinea pig & Millipore & AB5905 & RRID:AB_2301751 \\
\hline vGLuT3 & guinea pig & Synaptic Systems & 135204 & RRID:AB_2619825 \\
\hline vNUT & guinea pig & Millipore & ABN83 & n.a. \\
\hline \multicolumn{5}{|l|}{ Secondary antibodies } \\
\hline biotinylated anti-rabbit & donkey & BioRad & 644008 & RRID:AB_619842 \\
\hline DyLight 405 anti-mouse & donkey & Jackson & $715-475-150$ & RRID:AB_2340839 \\
\hline Alexa 405 anti-rabbit & donkey & Jackson & $711-475-152$ & RRID:AB_2340616 \\
\hline Alexa 488 anti-chicken & donkey & Jackson & 703-546-155 & RRID:AB_2340376 \\
\hline Alexa 488 anti-goat & donkey & Jackson & $705-546-147$ & RRID:AB_2340430 \\
\hline Alexa 488 anti-rabbit & donkey & Jackson & $711-546-152$ & RRID:AB_2340619 \\
\hline CF568 anti-mouse & donkey & Biotium & 20802 & RRID:AB_10853136 \\
\hline Alexa 594 anti-goat & donkey & LifeTech & A11058 & RRID:AB_2534105 \\
\hline
\end{tabular}




\begin{tabular}{|c|c|c|c|c|}
\hline Alexa 594 anti-guinea pig & goat & LifeTech & A11076 & RRID:AB_141930 \\
\hline Alexa 594 anti-mouse & donkey & LifeTech & A21203 & RRID:AB_141633 \\
\hline Alexa 594 anti-rabbit & donkey & LifeTech & A21207 & RRID:AB_141637 \\
\hline Alexa 594 anti-rat & donkey & Jackson & $712-585-150$ & RRID:AB_2340688 \\
\hline Alexa 647 anti-goat & donkey & Jackson & $705-606-147$ & RRID:AB_2340438 \\
\hline Alexa 647 anti-guinea pig & donkey & Jackson & $706-606-148$ & RRID:AB_2340477 \\
\hline Alexa 647 anti-mouse & donkey & Jackson & $715-605-150$ & RRID:AB_2340866 \\
\hline Alexa 647 anti-rabbit & donkey & Jackson & $711-605-152$ & RRID:AB_2492288 \\
\hline
\end{tabular}




\section{Movie S1.}

In vivo $2 \mathrm{P}$ time-lapse imaging shows temporal dynamics of microglia-neuron contacts. A tdTomato expressing neocortical neuron (red) is being contacted by processes of a microglial cell (green) in CX3CR1 1/GFP mouse electroporated in utero with PCAG-IRES-tdTomato. The analyzed trajectories of microglial processes contacting the neuron are shown on the right panel, warm colors label trajectories of somatic contacts, while cold colors label trajectories of microglial processes contacting neuronal dendrites. The middle panel shows the trajectories overlaid on the recording.

\section{Movie S2.}

In vitro CLSM time-lapse imaging of cocultured HEK293 and microglial cells. HEK cells were transfected with GFP-coupled control Kv2.1 construct on the left panel, and with YFP-coupled dominant-negative (DN) Kv2.1 construct on the right panel. Microglia is visualized by Alexa594-conjugated Lectin (red). Microglial processes contact Kv2.1-transfected HEK-cells at the clusters, but not those transfected with a dominant-negative mutant.

\section{Movie S3.}

Left: stack of electron tomographic $0.5 \mathrm{~nm}$ thick virtual sections shows the special nano-architecture of a somatic microglia-neuron junction with closely apposed mitochondria, mitochondria-associated membranes (MAMs) and cytoplasmatic structures. The silver-intensified P2Y12R-immunogold grains are clearly visible at the cytoplasmatic surface of microglial membrane. Note that large number of gold particles are clustered exactly where the neuronal cytoplasmatic structure is anchored. Right: 3D model of the same tomographic volume, neuronal membrane is magenta, microglial membrane is green, immunogold particles white, mitochondria light blue, MAM light green, cytoplasmatic densities red, vesicle-like structures blue and microglial reticular membrane structures darker green.

\section{Movie S4.}

Stack of electron tomographic virtual sections shows the special nano-architecture of the core of a somatic microglia-neuron junction. The silver-intensified P2Y12R-immunogold grains are clearly visible at the cytoplasmatic surface of microglial membrane. The tethers between the anchored mitochondria and MAM are clearly visible. The neuronal cytoplasmatic structure (mitochondria, MAM) is anchored to a membrane segment that is precisely facing the high density of P2Y12Rs on the microglial membrane. Note that distance between the neuronal and microglial membrane is the smallest exactly here, and intercellular tethers are also clearly visible.

\section{Movie S5.}

Left: stack of electron tomographic virtual sections shows the special nano-architecture of a somatic microglia-neuron junction with closely apposed mitochondria, MAMs and cytoplasmatic structures. The silver-intensified P2Y12R-immunogold grains are clearly visible at the cytoplasmatic surface of microglial membrane that touches the neuronal cell body, but are present only at a lower density at membrane segments, where the microglia touches a perisomatic bouton. Right: 3D model of the same tomographic volume, neuronal membrane is magenta, microglial membrane is ocker, immunogold particles white, mitochondria light blue, MAM light green, bouton membrane vivid green.

\section{Movie S6.}


In vivo $2 \mathrm{P}$ imaging of $\mathrm{CX} 3 \mathrm{CR} 1^{+/ G F P}$ mice in utero electroporated with CAG-Mito-R-Geco1 construct. Dashed lines show the outline of a neuron, green microglial processes touch neuronal cell body where somatic mitochondria (red) are present. White arrows indicate the contact sites of microglia.

\section{Movie S7.}

In vitro CLSM time-lapse imaging of KCl-stimulation of quinacrine-loaded cultured neuron. Left panel shows transmitted channel and superimposed green channel (quinacrine-labeled ATPcontaining vesicles), right panel shows the green channel and the outline of the neuron (white dashed line). White arrows point to vesicles that are released spontaneously, red arrows point to vesicles that are released after $40 \mathrm{mM} \mathrm{KCl} \mathrm{stimulation.} \mathrm{MIP} \mathrm{of} \mathrm{z-stack} \mathrm{(z-range:} 2.5 \mu \mathrm{m}$ ), frame dimension: $12.3 \times 22.2 \mu \mathrm{m}$. 